\title{
Lower Bounds on Trellis Complexity of Block Codes
}

\author{
Alec Lafourcade and Alexander Vardy, Senior Member, IEEE
}

\begin{abstract}
The trellis state-complexity $s$ of a linear block code is defined as the logarithm of the maximum number of states in its minimal trellis. We present a new lower bound on the statecomplexity of linear codes, which includes most of the existing bounds as special cases. The new bound is obtained by dividing the time axis for the code into several sections of varying lengths, as opposed to the division into two sections-the past and the future-employed in the well-known DLP bounds. For a large number of codes this results in a considerable improvement upon the DLP bound. Moreover, we generalize the new bound to nonlinear codes, and introduce several alternative techniques for lower-bounding the trellis complexity, based on the distance spectrum and other combinatorial properties of the code. We also show how the general ideas developed in this paper may be employed to lower-bound the maximum and the total number of branches in the trellis, leading to considerably tighter bounds on these quantities. Furthermore, the asymptotic behavior of the new bounds is investigated, and shown to improve upon the previously known asymptotic estimates of trellis state-complexity.
\end{abstract}

Index Terms-Trellis complexity, block codes, maximumlikelihood decoding, generalized Hamming weight hierarchy, dynamics of codes.

\section{INTRODUCTION}

$\mathbf{E}$ VERY linear block code may be represented by a trellis, which can be employed for maximum-likelihood softdecision decoding of the code with the Viterbi algorithm [9] or variants thereof. Although trellis description of block codes dates back to the work of Bahl et al. [1], Wolf [40], and Massey [28] in the 1970's, the subject has recently gained a considerable renewed interest [8], [10]--[14], [18]-[22], [25], [29], [30], [34], [35], [37], [42].

As shown in [10], [13], [22], [32], given an ordered time axis $\mathcal{I}=\{0,1, \cdots, n-1\}$, every linear block code $C$ has a unique minimal trellis, characterized by the fact that any other trellis for $C$ has at least as many states at time $i$ as the minimal trellis, for every $i \in \mathcal{I}$. Hence the trellis state-complexity $s$ of a linear code was defined in [11], [32] as

$$
s=\max _{i \in \mathcal{I}}\left\{\log _{q}\left|S_{i}\right|\right\}
$$

where $S_{i}$ is the set of states at time $i \in \mathcal{I}$ in the minimal trellis for $C$, and $q$ is the size of the code alphabet. Subsequently, other measures of trellis decoding complexity have been introduced, in particular the branch-complexity (cf. Forney [11])

Manuscript received December 16, 1994; revised April 21, 1995.This work was supported in part by the National Science Foundation under Grant NCR9409688. The material in this paper was presented in part at the 29th Annual Conference on Information Sciences and Systems, Baltimore, MD, March 1995.

The authors are with the Coordinated Science Laboratory, University of Illinois at Urbana-Champaign, Urbana, IL 61801 USA.

IEEE Log Number 9414733. and the edge-complexity-see McEliece [8], [29], [30]. The specific relations between the state-complexity $s$ and the branch and edge complexities are explored in-depth in Sections IV and $\mathrm{V}$ of this paper. IIere, we note that all these measures are closely related to (1), and refer to Muder [32] who claims $s$ to be a fundamental descriptive characteristic of the code, comparable to quantities such as length $n$, rate $R$, and minimum distance $d-$ a point of view shared by quite a number of recent works [10], [11], [14], [18], [19], [21], [22], [25], [34], [37], [41], [42].

Thus one of the main problems related to trellis representation of block codes is finding upper and lower bounds on state-complexity. Since the state-complexity of a given code $C$ depends on the ordering of the time axis $\mathcal{I}$ for $C$, the upper bounds are usually obtained by considering particular "good" permutations of the time axis [8], [11], [14], [19]-[21], [37], while the lower bounds follow by exploiting certain properties of the code that are invariant under coordinate permutations. Perhaps the earliest known lower bound on $s$ is due to Muder [32]: for an $(n, k, d)$ linear code

$$
s \geq k-\min _{i \in \mathcal{I}}\{K(i, d)+K(n-i, d)\}
$$

where $K(n, d)$ denotes the largest possible dimension of a lincar code of length $n$ and minimum Hamming distance $d$. The lower bound of (2) is based upon the relation

$$
s_{i} \stackrel{\text { def }}{=} \log _{q}\left|S_{i}\right|=k-p_{i}-f_{i}
$$

established in [10], where $p_{i}$ and $f_{i}$ stand for the dimension of the past subcode $\mathcal{P}_{i}$, respectively, future subcode $\mathcal{F}_{i}$, at time $i \in \mathcal{I}$. This implies

$$
s=k-\min _{i \in \mathcal{I}}\left\{p_{i}+f_{i}\right\}
$$

and Muder's bound of (2) follows immediately by observing that the minimum distance of both $\mathcal{P}_{i}$ and $\mathcal{F}_{i}$ is at least $d$. This bound was subsequently improved upon by several authors [19], [37], [11] who noticed that rather than just specifying a lower bound on the minimum distance of $\mathcal{P}_{i}$ and $\mathcal{F}_{i}$, it might be better to characterize $\mathcal{P}_{i}$ and $\mathcal{F}_{i}$ as subcodes of $C$ of support sizes $i$ and $n-i$. This yields

$$
s \geq k-\min _{i \in \mathcal{I}}\{k(i ; C)+k(n-i ; C)\}
$$

where $k(i ; C)$ is the maximum dimension of any subcode of $C$ of support size $i$ (cf. [11]). The function $k(\cdot ; C)$ is closely related to the generalized Hamming weight (GHW) hierarchy of the code, studied in a great number of recent papers-see [6], [16], [17], [38], [39] and references therein. The sequence $\{k(0 ; C), k(1 ; C), \cdots, k(n ; C)\}$, which is uniquely determined 
by the GHW of $C$, was termed the dimension-length profile (DLP) of $C$ by Forney [11], and hence the lower bound of (4) has become known as the DLP bound. The DLP bound was first employed by Kasami et al. [18], [19] to establish the state-complexity of Reed-Muller codes from the knowledge of their GHW [38]. Subsequently, Vardy and Be'ery [37] used the DLP bound to show that $s \geq k-1$ or $s \geq k-2$ whenever $d / n \geq 0.1$ or $d / n \geq 0.33$, respectively, and estimate the state-complexity of $\mathrm{BCH}$ codes. The results of [37] have been somewhat sharpened by Ytrehus in [41]. Other DLP bounds on trellis-complexity are given in the recent work in [8], [20].

All the lower bounds above are based on the common idea of dividing the time axis for the code into two sections-the past and the future, and then bounding the dimension of the resulting state-space using any of the known upper bounds on the dimension of the corresponding subcodes $\mathcal{P}_{i}$ and $\mathcal{F}_{i}$. In [25], [24] we have recently derived a conceptually quite different bound

$$
s \geq\left\lceil\frac{k(d-1)}{n}\right\rceil
$$

based on dividing the time axis into $\lceil n /(d-1)\rceil$ sections, and using the fact that there can be no parallel transitions in a trellis section of length less than $d$. While the bound of (5) is generally weaker than (2), (4) for moderate lengths, for $n \rightarrow \infty$ it is often much tighter than the asymptotic Muder bound [32], [42], especially for the high-rate codes.

In the next section we present a new lower bound on $s$, which includes all of (2), (4), and (5) as special cases. The new bound is obtained by partitioning the time axis for $C$ into several-that is, generally more than two-sections of varying lengths, and then selecting the partition which yields the best lower bound on $s$. For great many codes this results in a substantial improvement upon the DLP bound. In particular, we have applied the proposed technique to all the 8128 best known binary linear codes of length $\leq 128$, and obtained over 3400 improvements on (2) and (4), sometimes by as much as nine units. As a particular example we indicate that the lower bound $s \geq 12$ for the $(64,39,10) \mathrm{BCH}$ code given in [37, Table 4] is now tightened to $s \geq 13$. We have compiled a complete table ${ }^{1}$ summarizing our results for binary linear codes of length $\leq 128$. A small representative table with new lower bounds on $s$ for some 20 linear codes is presented in Section II.

Moreover, in Section III-A we generalize the lower bound of Section II to nonlinear codes described by one-to-one trellises. This requires the extension of the notion of DLP to nonlinear codes, which follows naturally from the discussion in Section III-A and allows us to state the lower bounds on $s$ in the same form for both linear and nonlinear codes. We also present in Section III-B several new techniques for lower-bounding the trellis-complexity of linear and nonlinear codes, based on the distance spectrum and other combinatorial properties of the code. These techniques are illustrated by proving that any trellis for the $(16,256,6)$ Nordstrom-Robinson code must have at least 96 states at positions 9 and 7 .

\footnotetext{
${ }^{1}$ For a copy of this table, send electronic mail to trellis@golay.csl.uiuc.edu.
}

In Section IV we consider lower bounds on the trellis branch-complexity $b=\max _{i \in \mathcal{I}} b_{i}$ where $b_{i}$ is the logarithm of the number of branches in the trellis section corresponding to time $i \in \mathcal{I}$. This measure of trellis-complexity was introduced by Forney in [11], who also noticed that $b=s$ or $b=s+1$ for linear codes. Thus any lower bound on $s$ is also a lower bound on $b$. In Section IV, however, we show how the statecomplexity bounds of Sections II and III-A can be translated into a lower bound on $b$ which is often tighter than the obvious statement $b \geq s$. The new bound on branch-complexity was again applied to all the best known binary linear codes of length $\leq 128$, yielding over 3300 improvements over the DLP bound ([11, Theorem 10$]$ ). Notably, in 2621 out of the 3300 cases, the lower bound on $b$ is strictly greater than the lower bound on $s$.

In Section $\mathrm{V}$ we derive lower bounds on the total number of branches in the trellis-the trellis edge-complexity $E(C)$ as defined by McEliece in [8], [30]. The bound presented in Section V-A follows by solving a nonlinear integer programming problem with linear constraints, which arise from the general relations between the values of $b_{i}$ derived in Section IV. This often produces considerably tighter estimates of $E(C)$ than those obtained using the existing bounds. For instance, for the $(64,39,10) \mathrm{BCH}$ code, the previously best known bound $E(C) \geq 161020$ (cf. [8, Table 1]) is improved to $E(C) \geq 274172$. In Section V-B we introduce alternative methods for bounding the trellis edge-complexity, based on the general ideas developed in Section III-B. These methods are illustrated by means of several examples. In particular, we deduce lower bounds on $E(C)$ for some of the codes considered in [8, Table 1], improving upon this table in 7 out of the possible 17 cases. This establishes, for instance, that the trellis given in [8] for the $(15,5,7) \mathrm{BCH}$ code is optimal. A representative table of the new lower bounds on edgecomplexity, as compared to the existing bounds, is presented at the end of Section $V$.

Finally, in Section VI we investigate the asymptotic implications of the new lower bounds for $n \rightarrow \infty$. It is shown in [25] that given any sequence of codes of increasing length $n$, with rate fixed at $R$ and relative minimum distance fixed at $d / n=\delta$, the state-complexity is bounded by

$$
c_{1} n \leq s \leq c_{2} n
$$

for some constants $c_{1}$ and $c_{2}$ independent of $n$. For $n \rightarrow \infty$ the results of [25] establish $c_{1} \geq \delta R$, while the paper of Zyablov and Sidorenko [42] shows that $c_{1} \geq R-R_{\max }(2 \delta)$, where $R_{\max }(\cdot)$ is the function describing the McEliece-Rodemich-Rumsey-Welch upper bound [31]. Herein we prove that for $n \rightarrow \infty$

$$
\begin{aligned}
\frac{s}{n} \geq \max \left\{R-R_{\max }(2 \delta), \frac{1}{2} R-\right. & \frac{1}{2} R_{\max }(3 \delta), \\
& \left.\cdots, \delta R-\delta R_{\max }(1)=\delta R\right\}
\end{aligned}
$$

thus bridging the gap between the asymptotic lower bounds of [42] and [25]. These results are illustrated in Fig. 1 for binary codes meeting the asymptotic Gilbert-Varshamov bound. The Gilbert-Varshamov bound (cf. [27, p. 557]) states that there 
$\varsigma$

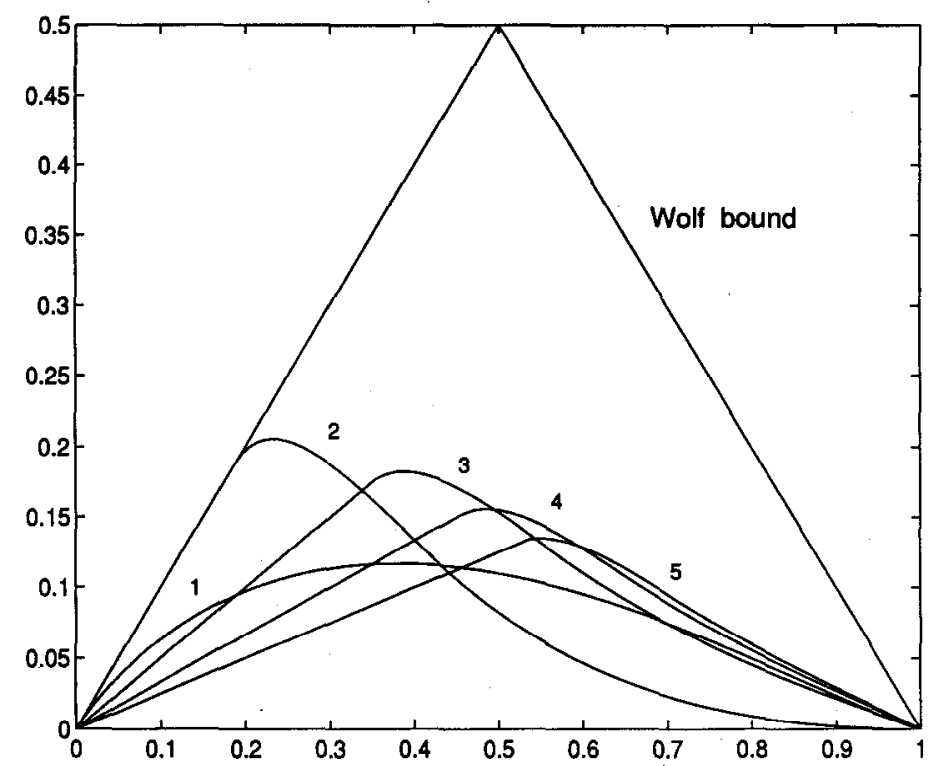

$R$

Fig. 1. Asymptotic lower bounds on trellis complexity for binary codes meeting the Gilbert-Varshamov bound. Curves labeled $2,3,4,5$ arise from Theorem 6.1 with $L=2,3,4,5$, respectively. The curve labeled 1 corresponds to $\varsigma \gtrsim 2 \delta R$ obtained by taking $L \simeq 1 / 2 \delta$.

$\varsigma$

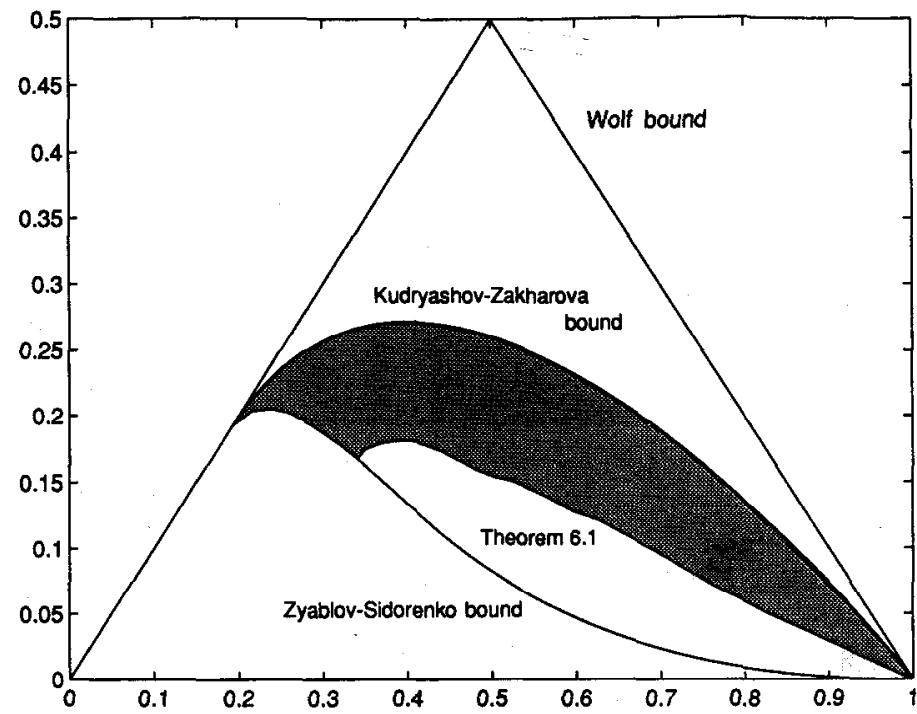

$R$

Fig. 2. Asymptotic trellis complexity of the best binary codes.

exist codes with $R \geq 1-H_{2}(\delta)$, where

$$
H_{2}(x)=x \log _{2} \frac{1}{x}+(1-x) \log _{2} \frac{1}{1-x}
$$

is the binary entropy function. The curve $R=1-H_{2}(\delta)$ describes the parameters $R$ and $\delta$ of the (asymptotically) best known binary codes. The next thing to consider is, perhaps, the trellis complexity. Fig. 2 illustrates the region where the parameters $R$ and $\varsigma=s / n$ of the best known binary codes must lie, and it may be seen from Fig. 2 that the new bounds on $\varsigma$ considerably narrow this region.

\section{BOUNDS ON STATE-COMPLEXITY OF LINEAR CODES}

We define a trellis $T$ for a block code as an edge-labeled directed graph $T=(S, A, E)$, where $S$ is the set of vertices or states, $A$ is the label alphabet of size $q$, and $E$ is the set of ordered triples $\left(\sigma, \sigma^{\prime}, \alpha\right)$ with $\sigma, \sigma^{\prime} \in S$ and $\alpha \in A$, called edges or branches. Furthermore, the set of states $S$ can he partitioned into disjoint subsets $S_{0}, S_{1}, \cdots S_{n}$ such that every branch $\left(\sigma, \sigma^{\prime}, \alpha\right)$ beginning at a state $\sigma \in S_{i}$ terminates at a state $\sigma^{\prime} \in S_{i+1}$, for all $i \in \mathcal{I}$. The subsets $S_{0}, S_{n} \subset S$ each consist of a single state, $S_{0}=\{\phi\}$ and $S_{n}=\{\varphi\}$ say, called the initial state and the final state, respectively. We assume that each state in the trellis lies on at least one path from $\phi$ 
to $\varphi$ (otherwise, this state may be removed without affecting the code described by $T$ ). Clearly, any path from $\phi$ to $\varphi$ in $T$ defines an $n$-tuple $\left(\alpha_{0}, \alpha_{1}, \cdots, \alpha_{n-1}\right)$ over $A$. We say that $T$ is a trellis realization of $C$, or simply a trellis for $C$, if the set of all such $n$-tuples is equal to the set of codewords of $C$.

In this section we let $C$ be a linear code over $\operatorname{GF}(q)$ of length $n$, dimension $k$, and minimum distance $d$. Further, we assume that the trellis $T$ for $C$ is proper (cf. [32]). For linear codes described by proper trellises, no two branches starting at the same state $\sigma \in S$ have the same label, which in particular implies that distinct paths from $\phi$ to $\varphi$ correspond to distinct codewords. Moreover [11], [13], the sets $S_{0}, S_{1}, \cdots, S_{n}$ may be identified with certain vector spaces over $\mathrm{GF}(q)$, which implies that $s_{i}=\log _{q}\left|S_{i}\right|$ is an integer for all $i$.

Given a state $\sigma \in S_{i}$ and an integer $0 \leq j \leq n-i$, let $P_{j}(\sigma)$ denote the set of all paths of length $j$ in $T$ starting at $\sigma$ (and terminating in one of the states of $S_{i+j}$ ). Further, given a particular $\sigma^{\prime} \in S_{i+j}$, we denote by $P\left(\sigma, \sigma^{\prime}\right)$ a subset of $P_{j}(\sigma)$ consisting of all the paths (of length $j$ ) in $T$ starting at $\sigma$ and terminating in $\sigma^{\prime}$. Clearly

$$
P_{j}(\sigma)=\bigcup_{\sigma^{\prime} \in S_{i+j}} P\left(\sigma, \sigma^{\prime}\right) .
$$

We have the following lemma.

Lemma 2.1: For any $\sigma \in S_{i}$ and $\sigma^{\prime} \in S_{i+j}$

$$
\log _{q}\left|P\left(\sigma, \sigma^{\prime}\right)\right| \leq k(j ; C)
$$

where $k(j ; C)$ is the $j$ th component of the dimension-length profile of $C$.

Proof: This essentially follows from the results of [11], [13]. We include the proof herein for completeness. First consider the path in $T$ corresponding to the all-zero codeword, and assume that both $\sigma$ and $\sigma^{\prime}$ lie on this path. Let

$$
\mathcal{J}=\{i, i+1, \cdots, i+j-1\} \subset \mathcal{I}
$$

and let

$C_{\mathcal{J}}=\left\{\underline{c}=\left(c_{0}, c_{1}, \cdots, c_{n-1}\right) \in C: c_{i}=0\right.$ for all $\left.: i \notin \mathcal{J}\right\}$

be the shortened subcode of $C$ whose support is confined to $\mathcal{J}$. Clearly, if $\sigma, \sigma^{\prime}$ lie on the path corresponding to the all-zero codeword, then any path in $P\left(\sigma, \sigma^{\prime}\right)$ can be completed to a codeword of $C_{\mathcal{J}}$. Since $|\mathcal{J}|=j$, we have $\operatorname{dim} C_{\mathcal{J}} \leq$ $k(j ; C)$ by the definition of $k(j ; C)$, and (7) follows. Now let $\sigma, \sigma^{\prime}$ be arbitrary states in $S_{i}$ and $S_{i+j}$, respectively, and distinguish between two cases. If there is no path from $\sigma$ to $\sigma^{\prime}$ in $T$ then $P\left(\sigma, \sigma^{\prime}\right)=\varnothing$ and (7) holds vacuously. Otherwise, let $\underline{c}^{*}=\left(c_{0}^{*}, c_{1}^{*}, \cdots, c_{n-1}^{*}\right)$ be a codeword of $C$ with $\left(c_{i}^{*}, c_{i+1}^{*}, \cdots c_{i+j-1}^{*}\right)$ corresponding to a path from $\sigma$ to $\sigma^{\prime}$ in $T$. A trellis $T^{*}$ for $\underline{c}^{*}+C$ may be obtained from $T$ by adding to the label of each branch the corresponding symbol of $c^{*}$. It is obvious that this does not alter the structure of the trellis, and in particular the number of paths from $\sigma$ to $\sigma^{\prime}$, and that $\sigma, \sigma^{\prime}$ lie on the all-zero path in $T^{*}$. (In other words, $P\left(\sigma, \sigma^{\prime}\right)$ is just a coset of $C_{\mathcal{J}}$, provided there exists a path from $\sigma \in S_{i}$ to $\sigma^{\prime} \in S_{i+j}$ ). Therefore, by the foregoing argument

$$
\log _{q}\left|P\left(\sigma, \sigma^{\prime}\right)\right| \leq k\left(j ; \underline{c}^{*}+C\right)=k(j ; C) .
$$

Lemma 2.1 in conjunction with (6) provides an upper bound on the total number of paths in $P_{j}(\sigma)$ for any $\sigma \in S_{i}$, as follows:

$$
\left|P_{j}(\sigma)\right|=\sum_{\sigma^{\prime} \in S_{i+j}}\left|P\left(\sigma, \sigma^{\prime}\right)\right| \leq\left|S_{i+j}\right| \cdot q^{k(j ; C)} .
$$

We now establish a relation between $\left|P_{j}(\sigma)\right|$ and the dimensions of the future subcodes of $C$ at times $i$ and $i+j$. This again follows easily from the results of Forney [11].

Lemma 2.2: For any $\sigma \in S_{i}$

$$
\log _{q}\left|P_{j}(\sigma)\right|=f_{i}-f_{i+j}
$$

Proof: It is known [11] that the number of branches beginning at a state $\sigma \in S_{i}$ is the same for all $\sigma \in S_{i}$, and is given by $\gamma_{i}=q^{f_{i}-f_{i \mid 1}}$ for all $i \in \mathcal{I}$. Therefore

$$
\left|P_{j}(\sigma)\right|=\prod_{l=i}^{i+j-1} \gamma_{l}=\prod_{l=i}^{i+j-1} q^{f_{l}-f_{l+1}}=q^{f_{i}-f_{i+j}}
$$

Using Lemma 2.2 in conjunction with (8), we obtain

$$
\begin{aligned}
s_{i+j} & =\log _{q}\left|S_{i+j}\right| \geq \log _{q}\left|P_{j}(\sigma)\right|-k(j ; C) \\
& =f_{i}-f_{i+j}-k(j ; C) .
\end{aligned}
$$

We note that (9) is a generalization of the relation $s_{i+j} \geq$ $f_{i}-f_{i+j}$ for $j \leq d-1$, which was already established in [25]. The following lower bound on the state-complexity of linear codes is, in a sense, a corollary of (9).

Theorem 2.3: Let $l_{1}, l_{2}, \cdots, l_{L}$ be any set of positive integers, with $l_{1}+l_{2}+\cdots+l_{L}=n$. Then

$$
s \geq\left\lceil\frac{k-k\left(l_{1} ; C\right)-k\left(l_{2} ; C\right)-\cdots-k\left(l_{L} ; C\right)}{L-1}\right\rceil .
$$

Proof: We partition the trellis into $L$ sections of lengths $l_{1}, l_{2}, \cdots, l_{L}$. For $j=0,1, \cdots, L$, let $\beta_{j}=\sum_{i=1}^{j} l_{i}$ denote the section boundaries, with the convention that $\beta_{0}=0$. Then (9) implies

$$
\sum_{j=1}^{L} s_{\beta_{j}} \geq \sum_{j=1}^{L}\left(f_{\beta_{j-1}}-f_{\beta_{j}}-k\left(l_{j} ; C\right)\right) .
$$

Note that at time $i=0$ the entire code lies in the future while at time $i=n$ the entire code lies in the past. Hence $f_{0}=k$ while $f_{n}=0$, and therefore

$$
\sum_{j=1}^{L}\left(f_{\beta_{j-1}}-f_{\beta_{j}}\right)=f_{0}-f_{n}=k .
$$

Further, since $S_{n}=\{\varphi\}$ we have $s_{\beta_{L}}=s_{n}=0$. Thus (11) can be rewritten as

$$
\sum_{j=1}^{L-1} s_{\beta_{j}} \geq k-\sum_{j=1}^{L} k\left(l_{j} ; C\right)
$$

To complete the proof, observe that $s_{\beta_{j}} \leq s$ for all $j$, and that $s$ must be an integer for lincar codes. 
Remark: It is known [10] that the state-complexity of the dual code $C^{\perp}$ is equal to that of the primal code $C$. In conjunction with Theorem 2.3 , this immediately implies

$s \geq\left\lceil\frac{(n-k)-k\left(l_{1} ; C^{\perp}\right)-k\left(l_{2} ; C^{\perp}\right)-\cdots-k\left(l_{L} ; C^{\perp}\right)}{L-1}\right\rceil$

for any positive $l_{1}, l_{2}, \cdots, l_{L}$, such that $l_{1}+l_{2}+\cdots+l_{L}=n$.

It is easy to see that the DLP bound of (4) is a special case of Theorem 2.3 obtained by setting $L=2$ and then maximizing over all the partitions of the type $l_{1}=i$ and $l_{2}=n-i$. The lower bound

$$
s \geq\left\lceil\frac{k(d-1)}{n}\right\rceil
$$

of [25] is also a special case of Theorem 2.3, obtained by taking $L=\lceil n /(d-1)\rceil$ and letting $l_{1}, l_{2}, \cdots, l_{L} \leq d-1$. In this case, obviously,

$$
k\left(l_{1} ; C\right)=k\left(l_{2} ; C\right)=\cdots=k\left(l_{L} ; C\right)=0
$$

and Theorem 2.3 reduces to (5).

We note that although the lower bound of Theorem 2.3 is stated in terms of the DLP function $k(\cdot ; C)$, the complete dimension-length profile is known for very few codes. If the DLP is not known for the code at hand, then $k(\cdot ; C)$ in Theorem 2.3 can be replaced by an upper bound thereupon as follows. Obviously, $k(i ; C) \leq K(i, d)$ for all $i \in \mathcal{I}$. Furthermore, it is shown in [11], [8] that

$$
k(i ; C)=i-(n-k)+k\left(n-i ; C^{\perp}\right)
$$

and therefore

$$
\begin{aligned}
k(i ; C) & \leq \min \left\{K(i, d), i-(n-k)+K\left(n-i, d^{\perp}\right)\right\} \\
& \leq K(i, d)
\end{aligned}
$$

We will use the first inequality in (13) if the dual distance $d^{\perp}$ is known, and resort to the second inequality, otherwise. Note that the latter approach essentially reduces the DLP bound to the Muder bound, in which case Theorem 2.3 becomes a generalization of (2).

Example 2.1: Consider the $(64,39,10) \mathrm{BCH}$ code. The GHW of this code is not known, and the dual distance is $d^{\prime}=8$. In this case, the DLP bound, with (13) used to evaluate $k(\cdot ; C)$, gives

$$
s \geq 39-K(31,10)-K(33,10) \geq 39-(13+14)=12
$$

However, partitioning into three sections of lengths $l_{1}=22$, $l_{2}=20$, and $l_{3}=22$, in conjunction with Theorem 2.3 and the Brouwer-Verkoeff table [5], we obtain

\begin{tabular}{|c|c|c|c|}
\hline \multirow{2}{*}{ Code } & \multicolumn{3}{|c|}{ Bounds on $s$} \\
\hline & $\begin{array}{l}\text { Upper } \\
\text { bound }\end{array}$ & $\begin{array}{l}\text { Lower } \\
\text { bound }\end{array}$ & Reference \\
\hline 1. $\mathrm{BCH}[8,4,4]$ & 3 & 3 & [19] \\
\hline 2. $\mathrm{BCH}[16,11,4]$ & 4 & 4 & [19] \\
\hline 3. $\mathrm{BCH}[16,7,6]$ & 6 & 6 & [41] \\
\hline 4. $\mathrm{BCH}[16,5,8]$ & 4 & 4 & [19] \\
\hline 5. $\mathrm{BCH}[32,26,4]$ & 5 & 5 & [19] \\
\hline 6. $\mathrm{BCH}[32,21,6]$ & 10 & 10 & {$[41]$} \\
\hline 7. $\mathrm{BCH}[32,16,8]$ & 9 & 9 & {$[19]$} \\
\hline 8. $\mathrm{BCH}[32,11,12]$ & 10 & 10 & [41] \\
\hline 9. $\mathrm{BCH}[32,6,16]$ & 5 & 5 & [19] \\
\hline 10. $\mathrm{BCH}[64,57,4]$ & 6 & 6 & [19] \\
\hline 11. $\mathrm{BCH}[64,51,6]$ & 12 & 12 & {$[41]$} \\
\hline 12. $\mathrm{BCH}[64,45,8]$ & 14 & 12 & $(13),[5]$ \\
\hline 13. $\mathrm{BCH}[64,39,10]$ & 20 & 13 & $(10),[5]$ \\
\hline 14. $\mathrm{BCH}[64,36,12]$ & 19 & 15 & {$[37]$} \\
\hline 15. ВCH $[64,30,14]$ & 21 & 16 & {$[37]$} \\
\hline 16. $\mathrm{BCH}[64,24,16]$ & 16 & 14 & [37] \\
\hline 17. $\mathrm{BCH}[64,18,22]$ & 17 & 17 & [41] \\
\hline 18. $\mathrm{BCH}[64,16,24]$ & 15 & 15 & {$[41]$} \\
\hline 19. $\mathrm{BCH}[64,10,28]$ & 9 & 9 & [37] \\
\hline 20. $\mathrm{BCH}[64,7,32]$ & 6 & 6 & [19] \\
\hline
\end{tabular}

$$
\begin{aligned}
s & \geq\left\lceil\frac{39-K(22,10)-K(20,10)-K(22,10)}{2}\right\rceil \\
& =\left\lceil\frac{39-5-4-5}{2}\right\rceil=13
\end{aligned}
$$

TABLE I

UPDATED BOUNDS ON TRELLIS STATE-COMPLEXITY for PRIMITIVE BINARY BCH CODES

Example 2.2: Now consider the $(127,85,13) \mathrm{BCH}$ code. Using the table of [5] to evaluate the DLP bound for this code we get $s \geq 14$. However, substituting the partition $l_{1}=l_{2}=l_{3}=30$, and $l_{4}=37$ into Theorem 2.3 produces

$$
\begin{aligned}
s & \geq\left\lceil\frac{85-3 \cdot K(30,13)-K(37,13)}{3}\right\rceil \\
& \geq\left\lceil\frac{85-3 \cdot 6-12}{3}\right\rceil=19 .
\end{aligned}
$$

Note that partition into four sections is necessary in this case—all the partitions with $L \neq 4$ yield bounds below $19 . \diamond$

Using (13) it is also possible to show that $s \geq 12$ for the $(64,45,8) \mathrm{BCH}$ code. An updated table of bounds on trellis complexity of all the extended primitive binary $\mathrm{BCH}$ codes of length $\leq 64$ is given Table I. Combining our results with those of Ytrehus [41], the original table of [37] is now improved in eight out of the eleven possible cases, leaving only five values of $s$ undetermined. These improvements are set in bold face. All the upper bounds in Table I are from [37].

Furthermore, we have applied the lower bound of Theorem 2.3 to all the 8128 best known binary linear codes of length $\leq 128$ in the table of Brouwer and Verhoeff [5]. This resulted in 3415 improvements upon the DLP bound, sometimes by as much as nine units. Due to space limitations, the complete table of our results is not included in this paper. ${ }^{2}$ Herein, we provide a small table-Table II-of lower

${ }^{2}$ It is available via electronic mail at trellis@golay.csl.uinc.edu 
TABLE II

LOWER BoundS ON TRELlis STATE-CompleXITY FOR SOME BINARY Linear CODES

\begin{tabular}{|c|c|c|c|}
\hline \multirow{2}{*}{ Code } & \multicolumn{3}{|c|}{ Bounds on $s$} \\
\hline & $\begin{array}{c}\text { DLP } \\
\text { bound }\end{array}$ & Partition & $\begin{array}{c}\text { New } \\
\text { bound }\end{array}$ \\
\hline 1. Hamming ${ }^{*}[9,3,4]$ & 1 & $\begin{array}{lll}3 & 3 & 3 \\
0 & 0 & 0\end{array}$ & 2 \\
\hline 2. Hamming ${ }^{*}[13,7,4]$ & 2 & $\begin{array}{lll}5 & 3 & 5 \\
1 & 0 & 1\end{array}$ & 3 \\
\hline 3. Shearer $*[36,21,7]$ & 7 & $\begin{array}{lll}12 & 12 & 12 \\
2 & 2 & 2\end{array}$ & 8 \\
\hline 4. Hamming* $[41,32,4]$ & 3 & $\begin{array}{lllll}9 & 9 & 5 & 9 & 9 \\
4 & 4 & 1 & 4 & 4\end{array}$ & 4 \\
\hline 5. $\mathrm{BCH}[64,39,10]$ & 12 & $\begin{array}{ccc}22 & 20 & 22 \\
5 & 4 & 5\end{array}$ & 13 \\
\hline 6. $\mathrm{BCH}[70,45,9]$ & 11 & $\begin{array}{ccc}24 & 22 & 24 \\
7 & 6 & 7\end{array}$ & 13 \\
\hline 7. $\mathrm{BCH}^{*}[73,46,10]$ & 11 & $\begin{array}{ccc}25 & 23 & 25 \\
7 & 6 & 7\end{array}$ & 13 \\
\hline 8. Shearer $*[73,50,8]$ & 7 & $\begin{array}{cccc}13 & 17 & 17 & 26 \\
2 & 5 & 5 & 12\end{array}$ & 9 \\
\hline 9. $\mathrm{BCH}[76,44,11]$ & 11 & $\begin{array}{ccc}27 & 22 & 27 \\
7 & 4 & 7\end{array}$ & 13 \\
\hline 10. $\mathrm{BCH}[76,50,9]$ & 10 . & $\begin{array}{ccc}24 & 28 & 24 \\
7 & 11 & 7\end{array}$ & 13 \\
\hline 11. Goppa* $[97,62,12]$ & 12 & $\begin{array}{ccc}28 & 41 & 28 \\
7 & 19 & 7\end{array}$ & 15 \\
\hline 12. Goppa $[105,56,16]$ & 13 & $\begin{array}{ccc}34 & 37 & 34 \\
6 & 8 & 6\end{array}$ & 18 \\
\hline 13. Goppa* $[109,61,14]$ & 7 & $\begin{array}{cccc}26 & 26 & 26 & 31 \\
3 & 3 & 3 & 6\end{array}$ & 16 \\
\hline 14. $\mathrm{BCH}[127,57,23]$ & 22 & $\begin{array}{ccc}42 & 43 & 42 \\
3 & 3 & 3\end{array}$ & 24 \\
\hline 15. $\mathrm{BCH}[127,64,21]$ & 22 & $\begin{array}{ccc}40 & 45 & 42 \\
3 & 6 & 4\end{array}$ & 26 . \\
\hline 16. $\mathrm{BCH}[127,71,19]$ & 21 & $\begin{array}{ccc}43 & 41 & 43 \\
7 & 6 & 7\end{array}$ & 26 \\
\hline 17. $\mathrm{BCH}[127,78,15]$ & 14 & $\begin{array}{lll}40 & 42 & 45 \\
11 & 13 & 15\end{array}$ & 20 \\
\hline 18. $\mathrm{BCH}[127,85,13]$ & 14 & $\begin{array}{cccc}30 & 30 & 30 & 37 \\
6 & 6 & 6 & 12\end{array}$ & 19 \\
\hline 19. $\mathrm{BCH}[127,92,11]$ & 13 & $\begin{array}{lll}42 & 43 & 42 \\
20 & 21 & 20\end{array}$ & 16 \\
\hline 20. $\mathrm{BCH}[127,99,9]$ & 12 & $\begin{array}{cccc}24 & 32 & 47 & 24 \\
7 & 14 & 28 & 7\end{array}$ & 15 \\
\hline
\end{tabular}

bounds on $s$ for 20 codes selected from the table of [5]. The values listed immediately below $l_{i}$ represent upper bounds on $k\left(l_{i} ; C\right)$, which are in most cases deduced from the table of [5]. The asterisk ${ }^{*}$ denotes shortening; the Shearer codes are from [33].

\section{LOWER BOUNDS FOR NONLINEAR CODES}

When dealing with nonlinear block codes, one needs to take special care since these codes do not necessarily possess a minimal trellis [22]. One way to avoid this problem is to consider only proper trellises for nonlinear codes, as defined by Muder [32]. However, this approach is too restrictive for our purposes. Instead, we shall limit our discussion to the oneto-one trellises, having the property that distinct paths through the trellis correspond to distinct codewords. Obviously, any proper trellis is one-to-one; for an example of one-to-one trellis which is not proper consider, for instance, [32, Fig. 1]. Given a nonlinear block code $C$ and an ordered time axis $\mathcal{I}$, the state-complexity of $C$ is defined [25] as

$$
s=\min _{T} \max _{i \in \mathcal{I}}\left\{\log _{q}\left|S_{i}\right|\right\}
$$

where the minimum is taken over all possible one-to-one trellises $T$ for $C$.

\section{A. Cardinality-Length Profile Bounds}

For our purposes, there are essentially two consequential differences between the linear and the nonlinear case. First, the dimension is no longer defined, so that the entities $k$ and $k\left(l_{1} ; C\right), k\left(l_{2} ; C\right), \cdots, k\left(l_{L} ; C\right)$, used in the lower bound of Theorem 2.3, lose their meaning. The first of these is naturally replaced by $\kappa=\log _{q}|C|$. To account for $k(\cdot ; C)$ we need to extend the notion of dimension-length profile to nonlinear codes. The "dimension" part is again naturally replaced by the log-cardinality. The notion of "length" is a little trickier, however. The "length" in the dimension-length profile has to do with the concept of support, which is, in turn, based on the assumption that the underlying alphabet has a group structure and contains a special element-zero. Yet, if the linearity is absent, there is nothing special about the zero element. Thus for nonlinear codes over an arbitrary alphabet (which need not have a group structure), we define effective length and variation-support as follows (see also [6]). Let $\mathcal{I}=\{0,1, \cdots, n-1\}$ be a time axis for a (nonlinear) code $C$. A position $i \in \mathcal{I}$ belongs to the variation-support of $C$ if and only if there exist codewords $\left(c_{0}, c_{1}, \cdots, c_{n-1}\right) \in C$ and $\left(c_{v}^{\prime}, c_{1}^{\prime}, \cdots, c_{n-1}^{\prime}\right) \in C$ with $c_{i} \neq c_{i}^{\prime}$. The effective length of $C$ is then the cardinality of its variation-support. With this notation, we have

Definition: Let $C$ be a (nonlinear) block code of length $n$ over an alphabet of size $q$. Then the cardinality-length profile of $C$ is given by the sequence

$$
\left\{\log _{q} M(0 ; C), \log _{q} M(1 ; C), \cdots, \log _{q} M(n ; C)\right\}
$$

where $M(l ; C)$ is the maximum cardinality of any subcode of $C$ of effective length $l$.

Note that for linear codes, the cardinality-length profile reduces to the DLP, which makes the foregoing definition a plausible generalization of the concept introduced by Forney in [11].

The second difficulty with nonlinear codes is that $\gamma_{i}$ in Lemma 2.2 cannot be in general expressed in terms of the cardinalities of the future subcodes and, in fact, the number of branches beginning at a given state in $S_{i}$ need not be the same for all $\sigma \in S_{i}$. The following theorem overcomes this problem by means of an enumerative argument, similar to the one employed in [25, proof of Theorem 5]. ${ }^{3}$

Theorem 3.1: For (nonlinear) block codes described by one-to-one trellises

$$
s \geq \frac{\log _{q}|C|-\sum_{j=1}^{L} \log _{q} M\left(l_{j} ; C\right)}{L-1}
$$

where $l_{1}, l_{2}, \cdots, l_{L}$ is any set of positive integers, such that $l_{1}+l_{2}+\cdots+l_{L}=n$.

Proof: As before, partition the one-to-one trellis $T$ for $C$ into $L$ sections of lengths $l_{1}, l_{2}, \cdots, l_{L}$, and for $j=0,1, \cdots, L$ let $\beta_{j}=\sum_{i=1}^{j} l_{i}$ denote the section boundaries. Further, for

\footnotetext{
${ }^{3}$ We are grateful to G. D. Forney, Jr. for his contribution to the proof of this theorem in [25].
} 
$j=1,2, \cdots, L$ define

$$
N_{j}=\frac{\left|P_{\beta_{j}}(\phi)\right|}{\left|P_{\beta_{j-1}}(\phi)\right|}
$$

with the convention that $\left|P_{\beta_{0}}(\phi)\right|=\left|P_{0}(\phi)\right|=1$. Thus $N_{j}$ is the ratio of the number of paths in the trellis terminating in $S_{\beta_{j}}$ to the number of paths terminating in $S_{\beta_{j-1}}$. Note that $\left|P_{\beta_{L}}(\phi)\right|=\left|P_{n}(\phi)\right|$ is the total number of paths in the trellis. Therefore, since the trellis is one-to-one, we have

$$
|C|=\left|P_{\beta_{L}}(\phi)\right|=\prod_{j=1}^{L} N_{j}
$$

Furthermore, by the definition of $N_{j}$, there must be at least $N_{j}$ paths from at least one state $\sigma^{*} \in S_{\beta_{j-1}}$ to any of the states in $S_{\beta_{j}}$, or in other words

$$
\left|P_{l_{j}}\left(\sigma^{*}\right)\right|=\sum_{\sigma \in S_{\beta_{j}}}\left|P\left(\sigma^{*}, \sigma\right)\right| \geq N_{j}
$$

for all $j=1,2, \cdots, L$. Now observe that for all $\sigma \in S_{\beta_{j}}$ we have

$$
\left|P\left(\sigma^{*}, \sigma\right)\right| \leq M\left(l_{j} ; C\right)
$$

since the paths in $P\left(\sigma^{*}, \sigma\right)$ can be completed to codewords of a subcode $C^{\prime} \subset C$, such that the variation-support of $C^{\prime}$ has size at most $l_{j}$. Combining this with (14), (15), and taking logarithms, we obtain

$$
\begin{aligned}
\log _{q}|C| & =\sum_{j=1}^{L} \log _{q} N_{j} \\
& \leq \sum_{j=1}^{L}\left(\log _{q}\left|S_{\beta_{j}}\right|+\log _{q} M\left(l_{j} ; C\right)\right) .
\end{aligned}
$$

Upon rearranging, (17) yields

$$
\sum_{j=1}^{L} s_{\beta_{j}} \geq \log _{q}|C|-\sum_{j=1}^{L} \log _{q} M\left(l_{j} ; C\right)
$$

similarly to (12). The theorem now follows by noting that $s_{\beta_{j}} \leq s$ for all $j$, and that $s_{\beta_{L}}=s_{n}=0$.

Indeed, if the minimum distance $d$ of $C$ is known, then the cardinality-length profile in the lower bound of Theorem 3.1 can he obviously upper-bounded hy $M(l ; C) \leq$ $A_{q}(l, d)$, where $A_{q}(n, d)$ denotes the largest possible cardinality of a code of length $n$ and minimum Hamming distance $d$ over an alphabet of size $q$. This reduces Theorem 3.1 to a generalization of Theorem 2 of Muder [32]. Whether there exists a bound on $M(l ; C)$ in terms of the dual distance of $C$, similar to (13), remains an open question. However we have

Proposition 3.2: Let $C$ be a code over $\mathrm{GF}(q)$ with dual distance $d^{\prime}$. Then for $l \geq n-d^{\prime}+1$

$$
\log _{q} M(l ; C)=\log _{q}|C|-(n-l) .
$$

Proof: It is known $[27$, p. 139] that $C$ is an orthogonal array of strength $d^{\prime}-1$. Thus every $n-l \leq d^{\prime}-1$ positions in $C$ contain each $q$-ary $(n-l)$-tuple exactly $|C| / q^{n-t}$ times, and the proposition follows.

Observe that for $l \geq n-d^{\prime}+1,(18)$ may be rewritten as

$$
\log _{q} M(l ; C)=l-\left(n-\log _{q}|C|\right)-\log _{q} A_{q}\left(n-l, d^{\prime}\right)
$$

which shows the analogy between Proposition 3.2 and (13). The difficulties in generalizing this beyond $l=n-d^{\prime}+1$ follow from the fact that $d^{\prime}$ is defined in terms of the MacWilliams transform of the distance spectrum of $C$, and hence a code with such a minimum distance does not have to exist.

Example 3.1: Consider the $\left(33,2^{22}, 5\right)$ nonlinear binary code, obtained by shortening the Preparata code of length 63 (cf.[27]). Applying to this code the CLP bound analogous to (4)-that is, partitioning into the past and future sections only, produces $s \geq 4.23$. However, partitioning into three sections of lengths $l_{1}=l_{2}=12$ and $l_{3}=9$, we obtain

$$
\begin{aligned}
s & \geq \frac{22-2 \log _{2} A_{2}(12,5)-\log _{2} A_{2}(9,5)}{2} \\
& =\frac{22-2.5-\log _{2} 6}{2}=4.707 .
\end{aligned}
$$

Example 3.2 The Nordstrom-Robinson code $\mathcal{N}_{16}$ is a wellknown $(16,256,6)$ nonlinear code. Using the table of $A_{2}(n, d)$ in $[7$, p. 248], we obtain the following upper bounds on the cardinality-length profile of $\mathcal{N}_{16}$

$$
\begin{array}{rcccccccccc}
l: & 1 & 2 & 3 & 4 & 5 & 6 & 7 & 8 & 9 & \\
\log _{2} M\left(l ; \mathcal{N}_{16}\right): & 0 & 0 & 0 & 0 & 0 & 1 & 1 & 1 & 2 & \\
l: & & 10 & 11 & 12 & 13 & 14 & 15 & 16 \\
\log _{2} M\left(l ; \mathcal{N}_{16}\right): & 2.59 & 3.59 & 4.59 & 5 & 6 & 7 & 8
\end{array}
$$

The CLP lower bound based on partition into two sections and (19) gives

$\begin{array}{cccccccccc}i: & 0 & 1 & 2 & 3 & 4 & 5 & 6 & 7 & 8 \\ s_{i}: & 0 & 1 & 2 & 3 & 3.41 & 4.41 & 4.41 & 5 & 6 \\ i: & 9 & 10 & 11 & 12 & 13 & 14 & 15 & 16 & \\ s_{i}: & 5 & 4.41 & 4.41 & 3.41 & 3 & 2 & 1 & 0 . & \end{array}$

However, proceeding as in Theorem 3.1, we can partition the trellis into three sections of lengths $l_{1}=4, l_{2}=8$, and $l_{3}=4$, to obtain

$$
s_{4}+s_{12} \geq \frac{8-2 \log _{2} M\left(4 ; \mathcal{N}_{16}\right)-\log _{2} M\left(8 ; \mathcal{N}_{16}\right)}{2}=7
$$

which shows that either $s_{4}$ or $s_{12}$ must be $\geq 3.5$. Furthermore, using Proposition 3.2 along with the fact that $d^{\prime}=6$ for this code (cf. [27, p. 141]), we can improve the bound of (19) on the cardinality-length profile of $\mathcal{N}_{16}$ as follows:

$$
\begin{array}{rccccccccc}
l: & 1 & 2 & 3 & 4 & 5 & 6 & 7 & 8 & 9 \\
\log _{2} M\left(l ; \mathcal{N}_{16}\right): & 0 & 0 & 0 & 0 & 0 & 1 & 1 & 1 & 2 \\
l: & 10 & 11 & 12 & 13 & 14 & 15 & 16 & & \\
\log _{2} M\left(l ; \mathcal{N}_{16}\right): & 2.59 & 3 & 4 & 5 & 6 & 7 & 8 & &
\end{array}
$$


This bound is obviously tight at all positions except possibly 9 and 10 . Using the construction of [36], it is easy to verify that indeed $\log _{2} M\left(10 ; \mathcal{N}_{16}\right)=\log _{2} 6$ as above; yet $\log _{2} M\left(9 ; \mathcal{N}_{16}\right)=\log _{2} 3 \leq 1.59$. This establishes the complete CLP of $\mathcal{N}_{16}$ and yields the following lower bounds on the state-complexity profile

$\begin{array}{cccccccccc}i: & 0 & 1 & 2 & 3 & 4 & 5 & 6 & 7 & 8 \\ s_{i}: & 0 & 1 & 2 & 3 & 4 & 5 & 4.41 & 5.41 & 6 \\ i: & 9 & 10 & 11 & 12 & 13 & 14 & 15 & 16 & \\ s_{i}: & 5.41 & 4.41 & 5 & 4 & 3 & 2 & 1 & 0 . & \end{array}$

The trellis for $\mathcal{N}_{16}$ constructed by Forney in [10] indeed has $2^{6}=64$ states at position 8 . However, all this fails to show that in fact $s \geq \log _{2} 96 \geq 6.58$ for $\mathcal{N}_{16}$.

\section{B. Bounds Based on Combinatorial Properties of the Code}

We now introduce several alternative techniques for lower bounding the state-complexity of both linear and nonlinear codes. These techniques will be illustrated by showing that a trellis for $\mathcal{N}_{16}$ must have at least 48 states at positions 6 and 10, and at least 96 states at positions 7 and 9. In what follows we consider only binary codes described by proper trellises, although most of the results easily extend to other cases as well. We generally distinguish between four types of states, depending upon the number of incoming and outgoing branches:

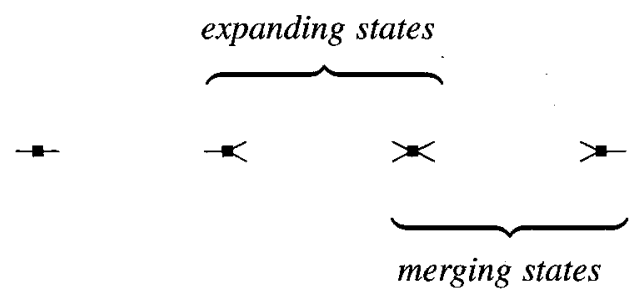

If the trellis is two-way proper-that is, it is proper when read from left to right and also when read from right to left, then these are the only possible types of states. This condition always holds for linear codes.

Proposition 3.3: If all the $2^{m}$ binary $m$-tuples appear at least once in every $m$ positions of $C$, then all the states in $S_{0}, S_{1}, \cdots, S_{m-1}$ are expanding and all the states in $S_{n-m+1}, S_{n-m+2}, \cdots, S_{n}$ are merging.

Proof: Replace the trellis with a binary tree. The condition of the proposition implies that the tree must have $2^{m}$ distinct nodes at depth $m$, which is only possible if all the states in $S_{0}, S_{1}, \cdots, S_{m-1}$ are expanding. A similar argument, with the trellis being read from right to left, shows that all the states in $S_{n-m+1}, S_{n-m+2}, \cdots, S_{n}$ must be merging.

Let $d^{\perp}$ denote the minimum distance of the linear code $C^{\perp}$ consisting of those $n$-tuples that are orthogonal to all the codewords of $C$. Note that in general $d^{\perp} \neq d^{\prime}$; for instance, for the Nordstrom-Robinson code $d^{\prime}=6$ while $d^{\perp}=8$ (cf. [36]). It is easy to see that the largest integer $m$ for which the condition of Proposition 3.3 can be satisfied is $m=d^{\perp}-1$. In fact, it can be verified that $\mathcal{N}_{16}$ attains this bound with equality. Thus in any proper trellis for $\mathcal{N}_{16}$ all the states in $S_{0}, S_{1}, \cdots, S_{6}$ must be expanding.

Proposition 3.4: None of the states in $S_{0}, S_{1}, \cdots, S_{d-1}$ is merging and none of the states in $S_{n-d+1}, S_{n-d+2}, \cdots, S_{n}$ is expanding.

Proof: Otherwise, there would be two codewords in $C$ at distance $\leq d-1$.

Propositions 3.3 and 3.4 establish that in any proper trellis for $\mathcal{N}_{16}$ all the states in $S_{0}, S_{1}, \cdots, S_{5}$ are of type $-<<$ and that $\left|S_{i}\right|=2^{i}$ for $i=0,1, \ldots, 5$. By Proposition 3.3, the states in $S_{6}$ could be either $><$ or $\rightarrow<$. Let us denote the number of states of type $><$ and $<$ by $a$ and $a^{\prime}$; respectively. Then, counting the number of branches beginning in $S_{5}$ and terminating in $S_{6}$, we obtain

$$
2 a+a^{\prime}=2\left|S_{5}\right|=64
$$

since each of the states in $S_{5}$ is expanding. Another relation between $a$ and $a^{\prime}$ follows from the next proposition.

Let $\left\{B_{0}, B_{1}, \cdots, B_{n}\right\}$. denote the distance spectrum of $C$. That is

$$
B_{i}=\frac{1}{|C|} \sum_{\underline{c} \in C} A_{i}(\underline{c})
$$

and $A_{i}(\underline{c})$ is the number of vectors of weight $i$ in $\underline{c}+C$. Define the distance set of $C$ as $D(C)=\left\{0 \leq i \leq n: B_{i} \neq 0\right\}$. Now let $\sigma \in S_{i}$, and consider the codes $\mathcal{P}_{\sigma}$ and $\mathcal{F}_{\sigma}$ corresponding to $P(\phi, \sigma)$ and $P(\sigma, \varphi)$, respectively. Clearly

$$
D\left(\mathcal{P}_{\sigma}\right) \subset\{0, d, d+1, \cdots, i\}
$$

and

$$
D\left(\mathcal{F}_{\sigma}\right) \subset\{0, d, d+1, \cdots, n-i\} .
$$

Furthermore, we have

Proposition 3.5:

$$
\begin{aligned}
& D\left(\mathcal{F}_{\sigma}\right) \subset D(C) \ominus D\left(\mathcal{P}_{\sigma}\right) \stackrel{\text { def }}{=} \bigcap_{j \in D\left(\mathcal{P}_{\sigma}\right)}\{w-j: w \in D(C)\} \\
& D\left(\mathcal{P}_{\sigma}\right) \subset D(C) \ominus D\left(\mathcal{F}_{\sigma}\right) \stackrel{\text { def }}{=} \bigcap_{j \in D\left(\mathcal{F}_{\sigma}\right)}\{w-j: w \in D(C)\} .
\end{aligned}
$$

Proof: Let $\underline{x}, \underline{x}^{\prime}$ be two codewords at distance $j$ in $\mathcal{P}_{\sigma}$ and let $\underline{y}, \underline{y}^{\prime}$ be two codewords at distance $j^{\prime}$ in $\mathcal{F}_{\sigma}$. Then $(\underline{x} \mid y)$ and $\left(\underline{x}^{\prime} \mid \underline{y}^{\prime}\right)$, where $(\cdot \mid \cdot)$ denotes concatenation, are two codewords in $C$ at distance $j+j^{\prime}$. It follows that $j^{\prime} \in\{w-j: w \in D(C)\}$.

Suppose that $\sigma$ is a state of type $>$ at position 6 in a trellis for $\mathcal{N}_{16}$. It is well-known [27, p. 741] that $D\left(\mathcal{N}_{16}\right)=$ $\{0,6,8,10,16\}$, and it is easy to see that $D\left(\mathcal{P}_{\sigma}\right)=\{0,6\}$. Hence, by Proposition 3.5, we have

$$
\begin{aligned}
D\left(\mathcal{F}_{\sigma}\right) & \subset D\left(\mathcal{N}_{16}\right) \Theta D\left(\mathcal{P}_{\sigma}\right) \\
& =\{0,6,8,10,16\} \cap\{-6,0,2,4,10\} \\
& =\{0,10\} .
\end{aligned}
$$

Since $\mathcal{F}_{\sigma}$ is of length 10 , this shows that $|P(\sigma, \varphi)|=\left|\mathcal{F}_{\sigma}\right|=2$. Note that this bound is considerably stronger than $|P(\sigma, \varphi)| \leq$ $M\left(10 ; \mathcal{N}_{16}\right)=6$ obtained using the CLP only. 
We can now establish a second relation between $a$ and $a^{\prime}$ as follows. For all $i=0,1, \cdots, n$, the total number of paths in the trellis is given by

$$
|C|=|P(\phi, \varphi)|=\sum_{\sigma \in S_{i}}|P(\phi, \sigma)| \cdot|P(\sigma, \varphi)| .
$$

Hence, for the Nordstrom-Robinson code

$$
\begin{aligned}
256 & =\sum_{\sigma \in S_{6}}|P(\phi, \sigma)| \cdot|P(\sigma, \varphi)| \\
& \leq a^{\prime} M\left(10 ; \mathcal{N}_{16}\right)+a\left|\mathcal{P}_{\sigma}\right|\left|\mathcal{F}_{\sigma}\right| \\
& =6 a^{\prime}+4 a .
\end{aligned}
$$

Solving for the minimum of $\left|S_{6}\right|=a+a^{\prime}$ subject to the constraints (20) and (22), we obtain $\left|S_{6}\right| \geq 16+32=48$. We are now ready to prove

Corollary 3.6: The state-complexity of a proper trellis for $\mathcal{N}_{16}$ is at least $s \geq \log _{2} 96$.

Proof: Using Proposition 3.5, it is easy to verify that for any $\sigma \in S_{7}$ either $|P(\phi, \sigma)|=1$ or $|P(\sigma, \varphi)|=1$. Therefore, $\sigma$ could not be of type $>$, and all the states in $S_{7}$ can be partitioned into the following categories:

Let $a_{1}$ denote the number of states of type $>-$; namely, those states in $S_{7}$ that follow a merge in $S_{6}$. Further, let $a_{2}$ be the number of states of type $>-$, and let $a_{3}$ be the number of states that are either of type - or of type $-<$. Then, counting the total number of branches between $S_{6}$ and $S_{7}$, and using the fact that all the states in $S_{6}$ are expanding, we obtain $a_{1}+2 a_{2}+a_{3}=2\left|S_{6}\right|$. Now, let $a$ be the number of merges in $S_{6}$, as in (20). Then we obviously have $\left|S_{6}\right|=64-a$ and $a_{1}=2 a$. Combining all of the above yields

$$
2 a_{1}+2 a_{2}+a_{3}=128 .
$$

Further, using (21) with $i=7$ to count the total number of paths in trellis, we get

$$
256 \leq 2 a_{1}+2 a_{2}+a_{3} M\left(9 ; \mathcal{N}_{16}\right)=2 a_{1}+2 a_{2}+3 a_{3} .
$$

Adding (23) and (24) yields $4\left(a_{1}+a_{2}+a_{3}\right)=4\left|S_{7}\right| \geq 384$, and hence $\left|S_{7}\right| \geq 96$.

Summarizing our results, we have the following lower bounds on the state-complexity of the Nordstrom-Robinson code:

$$
\begin{array}{rccccccccc}
i: & 0 & 1 & 2 & 3 & 4 & 5 & 6 & 7 & 8 \\
\left|S_{i}\right|: & 1 & 2 & 4 & 8 & 16 & 32 & 48 & 96 & 64 \\
& & & & & & & & & \\
i: & 9 & 10 & 11 & 12 & 13 & 14 & 15 & 16 \\
\left|S_{i}\right|: & 96 & 48 & 32 & 16 & 8 & 4 & 2 & 1
\end{array}
$$

Note that the bounds $\left|S_{10}\right| \geq 48$ and $\left|S_{9}\right| \geq 96$ follow by reading the trellis from left to right and applying arguments similar to the above. The trellis for $\mathcal{N}_{16}$ given by Forney in
[10] and the one arising from the construction of [36] coincide, yielding the state-complexity profile

$$
\begin{array}{rccccccccc}
i: & 0 & 1 & 2 & 3 & 4 & 5 & 6 & 7 & 8 \\
\left|S_{i}\right|: & 1 & 2 & 4 & 8 & 16 & 32 & 64 & 128 & 64 \\
i: & 9 & 10 & 11 & 12 & 13 & 14 & 15 & 16 & \\
\left|S_{i}\right|: & 128 & 64 & 32 & 16 & 8 & 4 & 2 & 1 &
\end{array}
$$

with no merges in $S_{6}$ and $S_{7}$. We conjecture that this profile is optimal componentwise.

\section{LOWER BOUNDS ON BRANCH COMPLEXITY}

For Viterbi decoding, the total number of trellis branches per unit time is usually regarded as a more accurate measure of decoding complexity than the (maximum) number of states in the trellis. In [11] Forney shows that for a linear code $C$ over $\mathrm{GH}(q)$, the set of branches

$$
\mathcal{B}_{i} \stackrel{\text { def }}{=}\left\{\left(\sigma, \sigma^{\prime}, \alpha\right): \sigma \in S_{i}, \sigma^{\prime} \in S_{i+1}, \alpha \in \mathrm{GF}(q)\right\}
$$

is a linear space, termed the branch space, for all $i \in \mathcal{I}=$ $\{0,1, \cdots, n-1\}$. He then defines the branch-complexity of $C$ as $b=\max _{i \in \mathcal{I}} b_{i}$, where $b_{i}$ is the dimension of the branch space at time $i$. This definition may be easily extended to nonlinear codes as well, by taking $b_{i}$ as the logarithm of the number of branches in the trellis at time $i$.

The following observation is immediate: for proper trellises $s_{i} \leq b_{i} \leq s_{i}+1$ since each state must have at least one outgoing branch and, in a proper trellis, cannot have more than $q=|A|$ outgoing branches. Thus for linear codes $b=s$ or $b=s+1$, and any lower bound on $s$ is also a lower bound on $b$. Nevertheless, it is reasonable to ask whether Theorem 2.3 can be modified into a lower bound on $b$ which is tighter than the obvious statement $b \geq s$. Such a lower bound is derived in what follows.

We start with a simple observation ([11, Lemma 5]): for linear codes $b_{i}=k-p_{i}-f_{i+1}$. This in conjunction with (3) implies

$$
b_{i}=s_{i}+f_{i}-f_{i+1} .
$$

Substituting this into (9), we conclude that for all $i \in \mathcal{I}$ and all $0 \leq j \leq n-i-1$, the dimension of the branch space at time $i+j$ is lower-bounded by

$$
b_{i+j} \geq f_{i}-f_{i+j+1}-k(j ; C) .
$$

This immediately leads to a lower bound on branch-complexity analogous to Theorem 2.3 .

Theorem 4.1: Let $l_{1}, l_{2}, \cdots l_{L}$ be positive integers, such that $l_{1}+l_{2}+\cdots+l_{L}=n-L+1$. Then

$$
b \geq\left\lceil\frac{k-k\left(l_{1} ; C\right)-k\left(l_{2} ; C\right)-\cdots-k\left(l_{L} ; C\right)}{L-1}\right\rceil .
$$


Proof: As in Theorem 2.3, we partition the trellis into $L$ sections. However, the section boundaries are now defined as follows:

$$
\begin{aligned}
& \beta_{0}=0, \quad \beta_{1}=l_{1}, \quad \beta_{2}=\beta_{1}+l_{2}+1 \\
& \beta_{3}=\beta_{2}+l_{3}+1, \quad \cdots, \quad \beta_{L}=\beta_{L-1}+l_{L}+1 .
\end{aligned}
$$

The condition $l_{1}+l_{2}+\cdots+l_{L}=n-L+1$ then implies $\beta_{L}=n$. Furthermore, from (26) we obtain

$$
\begin{aligned}
& b_{\beta_{1}} \geq \int_{\beta_{0}}-\int_{\beta_{1}+1}-k\left(l_{1} ; C\right) \\
& b_{\beta_{j}} \geq f_{\beta_{j-1}+1}-f_{\beta_{j}+1}-k\left(l_{j} ; C\right), \\
& \quad \text { for } j=2,3, \cdots, L-1 .
\end{aligned}
$$

Using (9), along with $\beta_{L}=\left(\beta_{L-1}+1\right)+l_{L}=n$, yields

$$
0=s_{\beta_{L}} \geq f_{\beta_{L-1}+1}-f_{\beta_{L}}-k\left(l_{L} ; C\right) .
$$

Summing the $L$ inequalities (28) and (29) produces

$$
\begin{aligned}
\sum_{j=1}^{L-1} b_{\beta_{j}} & \geq f_{\beta_{0}}-f_{\beta_{L}}-\sum_{j=1}^{L} k\left(l_{j} ; C\right) \\
& =k-\sum_{j=1}^{L} k\left(l_{j} ; C\right) .
\end{aligned}
$$

Since $b$ is an integer and $b \geq b_{\beta_{j}}$ for all $j$, this completes the proof of the theorem.

Remark: Observe that $k(l-1 ; C) \geq k(l ; C)-1$, which implies that the RHS of (27) cannot exceed the RHS of (10) by more than 1 . This, of course, is to be expected from the fact that $b \leq s+1$.

We note that Theorem 4.1 may be also extended to nonlinear codes, along the lines of Theorem 3.1. Thus with $\log _{q} M(\cdot ; C)$ defined as in Section II, we have

Theorem 4.2: For (nonlinear) block codes described by one-to-one trellises

$$
b \geq \frac{\log _{q}|C|-\sum_{j=1}^{L} \log _{q} M\left(l_{j} ; C\right)}{L-1}
$$

where $l_{1}, l_{2}, \cdots, l_{L}$ is any set of positive integers, such that $l_{1}+l_{2}+\cdots+l_{L}=n-L+1$.

The proof of this essentially consists of the combination of the ideas of Theorem 4.1 and Theorem 3.1. We define the section boundaries as in Theorem 4.1, and let

$$
\begin{aligned}
& N_{1}=\frac{\left|P_{\beta_{1}+1}(\phi)\right|}{\left|P_{\beta_{0}}(\phi)\right|} \quad N_{L}=\frac{\left|P_{\beta_{L}}(\phi)\right|}{\left|P_{\beta_{L-1}+1}(\phi)\right|} \\
& N_{j}=\frac{\left|P_{\beta_{j}+1}(\phi)\right|}{\left|P_{\beta_{j-1}+1}(\phi)\right|}, \quad \text { for } j=2,3, \cdots, L-1 .
\end{aligned}
$$

Then for $j=2,3, \cdots, L-1$ there must be at least one state $\sigma^{*} \in S_{\beta_{j-1}+1}$ such that

$$
N_{j} \leq\left|P_{l_{j}+1}\left(\sigma^{*}\right)\right|=\sum_{\sigma \in S_{\beta_{j}+1}}\left|P\left(\sigma^{*}, \sigma\right)\right|=\sum_{\epsilon \in \mathcal{B}_{\beta_{j}}}\left|P\left(\sigma^{*}, \epsilon\right)\right|
$$

where $P\left(\sigma^{*}, \epsilon\right)$ is the set of paths in the trellis starting at $\sigma^{*} \in S_{\beta_{j-1}+1}$ and containing the branch $\epsilon \in \mathcal{B}_{\beta_{j}}$. Since $\epsilon$ is fixed, the effective length of $P\left(\sigma^{*}, \epsilon\right)$ is at most $l_{j}$ rather than $l_{j}+1$, and therefore $\left|P\left(\sigma^{*}, \epsilon\right)\right| \leq M\left(l_{j} ; C\right)$ as in (16). Hence

$$
\log _{q} N_{j} \leq b_{\beta_{j}}+\log _{q} M\left(l_{j} ; C\right)
$$

and the rest of the proof easily follows.

Example 4.1: Consider the $(103,81,8)$ code obtained by shortening the $(129,107,8)$ Goppa code. The DLP bounds on the state and branch complexity of this code are $s \geq 11$ and $b \geq 11$, respectively. Note that for this code Theorem 2.3 produces $s \geq 11$ as well, so we cannot improve our estimate of the state-complexity by partitioning into more than two sections. However, we can improve the bound on branch complexity using Theorem 4.1 and taking three sections of lengths $l_{1}=l_{3}=26, l_{3}=49$, with $l_{1}+l_{2}+l_{3}=101=n-2$. The resulting lower bound on $b$ is

$$
\begin{aligned}
b & \geq\left\lceil\frac{81-2 \cdot K(26,8)-K(49,8)}{3}\right\rceil \\
& \geq\left\lceil\frac{81-2 \cdot 12-33}{2}\right\rceil=12 .
\end{aligned}
$$

We have applied the lower bound of Theorem 4.1 to all the 8128 binary linear codes of length $\leq 128$ in the table of Brouwer and Verhoeff [5]. This again resulted in a large number of improvements ${ }^{4}$ over the DLP bound on branch complexity (Theorem 10 of Forney [11]). Specifically, Theorem 4.1 is tighter than the DLP bound in 3308 cases, sometimes by as much as nine units. We note that in 2621 out of the 3308 cases, the bound on $b$ of Theorem 4.1 is strictly greater than the bound on $s$ of Theorem 2.3. A representative list of lower hounds on $b$ for some 20 codes, along with the corresponding partitions $l_{1}, l_{2}, \cdots, l_{L}$, is given in Table III. Note that for the 12 codes included in both Tables II and III, the lower bounds on $s$ and $b$ do not coincide.

\section{LOWER BOUNDS ON EDGE-COMPLEXITY}

In a recent paper [30], McEliece studied the computational complexity of a generalized version of the Viterbi algorithm on a trellis, and concluded that this complexity is proportional to the total number of branches-or edges - in the trellis. Motivated by this result, he introduced

$$
E(C) \stackrel{\text { def }}{=} \sum_{i \in \mathcal{I}} q^{b_{i}}
$$

as the appropriate measure of trellis complexity. To quote [30], "...we encourage future researchers in this area to take the edge count as the measure of trellis complexity."

Although both $E(C)$ and $b=\max _{i \in \mathcal{I}} b_{i}$ have to do with the number of branches in the trellis, these quantities are obviously not the same. Since the term "branch-complexity" was already used by Forney [11] to denote $\max _{i \in \mathcal{I}} b_{i}$, we will hereafter refer to $E(C)$ as the edge-complexity of the trellis.

\footnotetext{
${ }^{4}$ These branch-complexity bounds are also available via e-mail at trellis@golay.csl.uiuc.edu.
} 
TABBLE III

Lower Bounds on Trellis Branch-Complexity FOR SOME BINARY Linear CODES

\begin{tabular}{|c|c|c|c|}
\hline \multirow{2}{*}{ Code } & \multicolumn{3}{|c|}{ Bounds on $b$} \\
\hline & $\underset{\text { bound }}{\text { DLP }}$ & Partition & $\begin{array}{l}\text { New } \\
\text { bound }\end{array}$ \\
\hline 1. Hamming*[18,10,4] & 2 & $\begin{array}{lll}5 & 6 & 5 \\
1 & 2 & 1\end{array}$ & 3 \\
\hline 2. Shearer $* 32,18,6]$ & 5 & $\begin{array}{ccc}10 & 10 & 10 \\
2 & 2\end{array}$ & 6 \\
\hline 3. Shearer $*[44,29,6]$ & 6 & $\begin{array}{ccc}10 & 13 & 19 \\
2 & 4 & 9\end{array}$ & 7 \\
\hline 4. $\mathrm{BCH}^{*}[73,46,10]$ & 12 & $\begin{array}{ccc}19 & 25 & 27 \\
3 & 7 & 9\end{array}$ & 14 \\
\hline 5. Shearer ${ }^{*}[73,50,8]$ & 8 & $\begin{array}{lll}26 & 19 & 26 \\
12 & 7 & 12\end{array}$ & 10 \\
\hline 6. $\mathrm{BCH}[76,44,11]$ & 12 & $\begin{array}{ccc}25 & 24 & 25 \\
6 & 5 & 6\end{array}$ & 14 \\
\hline 7. $\mathrm{BCH}[76,50,9]$ & 11 & $\begin{array}{ccc}25 & 24 & 25 \\
8 & 7 & 8\end{array}$ & 14 \\
\hline 8. Shearer* $[78,63,6]$ & 9 & $\begin{array}{lll}25 & 26 & 25 \\
14 & 15 & 14\end{array}$ & 10 \\
\hline 9. Alternant $*(82,55,9]$ & 11 & $\begin{array}{ccc}24 & 32 & 24 \\
7 & 14 & 7\end{array}$ & 14 \\
\hline 10. Hamming ${ }^{*}[92,46,15]$ & 15 & $\begin{array}{ccc}26 & 28 & 36 \\
2 & 3 & 8\end{array}$ & 17 \\
\hline 11. Goppa* $[97,62,12]$ & 13 & $\begin{array}{ccc}28 & 33 & 34 \\
7 & 11 & 12\end{array}$ & 16 \\
\hline 12. Goppa* $[103,81,8]$ & 11 & $\begin{array}{lll}26 & 49 & 26 \\
12 & 33 & 12\end{array}$ & 12 \\
\hline 13. Goppa $[105,56,16]$ & 14 & $\begin{array}{ccc}37 & 29 & 37 \\
8 & 3 & 8\end{array}$ & 19 \\
\hline 14. Goppa* $[115,87,9]$ & 12 & $\begin{array}{llll}24 & 32 & 32 & 24 \\
7 & 14 & 14 & 7\end{array}$ & 15 \\
\hline 15. Goppa* $[126,110,6]$ & 10 & $\begin{array}{lll}39 & 46 & 39 \\
27 & 34 & 27\end{array}$ & 11 \\
\hline 16. $\mathrm{BCH}[127,57,23]$ & 23 & $\begin{array}{ccc}43 & 39 & 43 \\
3 & 2 & 3\end{array}$ & 25 \\
\hline 17. $\mathrm{BCH}[127,78,15]$ & 15 & $\begin{array}{lll}40 & 45 & 40 \\
11 & 15 & 11\end{array}$ & 21 \\
\hline 18. $\mathrm{BCH}[127,85,13]$ & 14 & $\begin{array}{cccc}30 & 30 & 30 & 34 \\
6 & 6 & 6 & 9\end{array}$ & 20 \\
\hline 19. ВCH $[127,92,11]$ & 14 & $\begin{array}{lll}42 & 51 & 32 \\
20 & 28 & 11\end{array}$ & 17 \\
\hline 20. ВCH $[127,99,9]$ & 13 & $\begin{array}{llll}32 & 32 & 32 & 28 \\
14 & 14 & 14 & 11\end{array}$ & 16 \\
\hline
\end{tabular}

In [8], [20] the authors use the following lower bound on the edge-complexity of linear codes:

$$
E(C) \geq \sum_{i=0}^{n-1} q^{k-k(i ; C)-k(n-i-1 ; C)}
$$

which follows immediately from the relation $b_{i}=k-p_{i}-f_{i+1}$. This, along with (13) which may be used to upper-bound $k(\cdot ; C)$, is presently the best known general bound on $E(C)$. Since this bound essentially amounts to summing up the DLP bounds on $b_{i}$ at each position, we will refer to (31) as the DLP bound on edge-complexity.

Herein we show how the techniques developed in the previous sections to lower-bound $s$ and $b$ can be translated into powerful lower bounds on $E(C)$, which are often considerably tighter than the DLP bound of (31).

\section{A. Constraints on Edge-Complexity by Trellis Partitioning}

We start by observing that (30) in the proof of Theorem 4.1 can be converted into the following statement. Let $\mathcal{J}=\left\{j_{1}, j_{2}, \cdots, j_{L}\right\}$ be any subset of $\mathcal{I}=\{0,1, \cdots, n-1\}$. Assume without loss of generality that $j_{1}<j_{2}<\cdots<j_{L}$ and define the function

$$
\begin{aligned}
Q(\mathcal{J})= & Q\left(j_{1}, j_{2}, \cdots, j_{L}\right) \\
\stackrel{\text { def }}{=} & k-k\left(j_{1} ; C\right)-k\left(j_{2}-j_{1}-1 ; C\right) \\
& -\cdots-k\left(n-j_{L}-1 ; C\right) .
\end{aligned}
$$

Then, we have

\section{Proposition 5.1:}

$$
b_{j_{1}}+b_{j_{2}}+\cdots+b_{j_{L}} \geq Q\left(j_{1}, j_{2}, \cdots, j_{L}\right) .
$$

Proof: Follows immediately from (30).

Thus for any subset $\mathcal{J} \subset \mathcal{I}$, Proposition 5.1 produces a linear constraint on the values of $\left\{b_{j}: j \in \mathcal{J}\right\}$. We can therefore set up a nonlinear integer programming problem with linear constraints as follows:

MINIMIZE $F\left(b_{0}, b_{1}, \ldots, b_{n-1}\right) \stackrel{\text { def }}{=} q^{b_{0}}+q^{b_{1}}+\cdots+q^{b_{n-1}}$

SUBJECT TO

$$
\sum_{j \in \mathcal{J}} b_{j} \geq Q(\mathcal{J}), \quad \text { for all } \mathcal{J} \subset \mathcal{I}
$$

Let $F^{*}\left(b_{0}, b_{1}, \cdots, b_{n-1}\right)$ denote the optimal solution to the problem above. Then, by the definition of $E(C)$, we have

Theorem 5.2:

$$
E(C) \geq F^{*}\left(b_{0}, b_{1}, \cdots, b_{n-1}\right) .
$$

Note that the DLP bound of (31) is a special case of Theorem 5.2, which results when the constraints in the problem (*) are restricted to the $\left(\begin{array}{l}n \\ 1\end{array}\right)$ subsets $\mathcal{J}=\{j\}$ of cardinality 1 . The definition of $Q(\mathcal{J})$ then reduces to

$$
Q(j)=k-k(j ; C)-k(n-j-1 ; C)
$$

and, since all the $\left(\begin{array}{l}n \\ 1\end{array}\right)$ constraints ${ }^{5}$ are disjoint in this case, the optimal solution to $(*)$ is clearly given by the right-hand side of (31).

In general, however, we have $2^{n}$ different constraints, so that complete evaluation of the lower bound of Theorem 5.2 appears to be intractable. To overcome this problem we proceed as follows. The constraint $\mathcal{J} \subset \mathcal{I}$ is said to be useful if

$$
Q(\mathcal{J})>\sum_{j \in \mathcal{J}} Q(j)=\sum_{j \in \mathcal{J}} k-k(j ; C)-k(n-j-1 ; C) .
$$

Those constraints that are not useful may be safely discarded from $(*)$, since such constraints cannot lead to an improvement over the DLP bound. In all the cases that we have considered, this approach easily reduces the $2^{n}$ constraints in $(*)$ to a tractable number.

The remaining difficulty is to solve the nonlinear minimization problem $(*)$. This may be accomplished using standard (nonlinear) constrained optimization techniques-see, for instance, Bertsekas [4] and references therein. However, the following simple observations often suffice to find the optimal solution to $(*)$.

Given a constraint $\mathcal{J}$ which is disjoint with all the other constraints, it is obvious that setting $b_{j}=\lfloor Q(\mathcal{J}) /|\mathcal{J}|\rfloor$ or $b_{j}=\lceil Q(\mathcal{J}) /|\mathcal{J}|\rceil$ for all $j \in \mathcal{J}$ so that

$$
\sum_{j \in \mathcal{J}} b_{j}=Q(\mathcal{J})
$$

\footnotetext{
5 To simplify the terminology, we will hereafter use "constraint" and "subset" interchangeably, meaning that the constraints in $(*)$ are identified with the subsets of $\mathcal{I}$ that are used to generate them.
} 
minimizes the objective function $F\left(b_{0}, b_{1}, \cdots, b_{n-1}\right)$. Now, consider two partially overlapping constraints of the form

$$
\begin{aligned}
& b_{j}+b_{j_{1}} \geq a_{1} \\
& b_{j}+b_{j_{2}} \geq a_{2}
\end{aligned}
$$

which are disjoint with all the other useful constraints. Then clearly $F\left(b_{0}, b_{1}, \cdots, b_{n-1}\right)$ is minimized by requiring equality in (32), or in other words, setting $b_{j_{1}}=a_{1}-b_{j}$ and $b_{j_{2}}=$ $a_{2}-b_{j}$. The value of $b_{j}$ may then be determined by solving

$$
\frac{d}{d b_{j}}\left(q^{b_{j}}+q^{a_{1}-b_{j}}+q^{a_{2}-b_{j}}\right)=0
$$

which yields $b_{j}=\left\lceil\frac{1}{2} \max \left(a_{1}, a_{2}\right)\right\rceil$. This argument easily generalizes. That is, whenever we can identify a set of constraints $\mathcal{J}_{1}, \mathcal{J}_{2}, \cdots, \mathcal{J}_{m}$ which is disjoint with all the other constraints, and is such that there exists an integer solution to the system of equations

$$
\sum_{j \in \mathcal{J}_{\ell}} b_{j}=Q\left(\mathcal{J}_{\ell}\right), \quad \text { for } \ell=1,2, \cdots, m
$$

then the function $F\left(b_{0}, b_{1}, \cdots, b_{n-1}\right)$ is minimized by solving (33), and then possibly taking derivatives to optimize over the degree of freedom in (33).

Example 5.1: Consider the $(64,39,10) \mathrm{BCH}$ code with dual distance $d^{\perp}=8$. Note that for this code Theorem 4.1 coincides with the DLP bound

$$
b_{i} \geq 39-k(i ; C)-k(63-i ; C), \quad \text { for } i=0,1, \cdots, 63
$$

giving $b=\max _{i \in \mathcal{I}} b_{i} \geq 13$. Thus in this case, we cannot improve upon the DLP bound on the maximum branch complexity in the trellis. We can, however, substantially tighten the DLP bound on the total number of branches in the trellis - the edge-complexity. Using (13) to evaluate the DLP bound for the entire branch-complexity profile produces the values of $b_{i}$ given in Table IV, and the corresponding bound on edgecomplexity is $E(C) \geq 161020$, which coincides with the figure reported for this code in [8, Table I]. However, applying Theorem 5.2 we find 324 useful constraints, corresponding to partitions into three and four sections. A system of 20 inequalities extracted from these 324 constraints is given in Table V. This system, augmented by the 64 inequalities in (34), may be easily solved using the arguments outlined above. The solution produces the values of $b_{0}, b_{1}, \cdots, b_{63}$ that satisfy the constraints of Table V and (34), and minimize the objective function

$$
F\left(b_{0}, b_{1}, \cdots, b_{63}\right)=2^{b_{0}}+2^{b_{1}}+\cdots+2^{b_{63}} .
$$

These values are listed in Table VI, with the entries exceeding the DLP bound of (34) set in boldface. The resulting lower bound on edge-complexity is $E(C) \geq 274172$, which is about 1.5 times greater than the DLP bound.
TABLE IV

\begin{tabular}{|c|c|c|}
\hline $\begin{array}{l}b_{22}+b_{42} \geq 26 \\
b_{23}+b_{43} \geq 26 \\
b_{24}+b_{44} \geq 26 \\
b_{25}+b_{45} \geq 26 \\
b_{26}+b_{46} \geq 26 \\
b_{36}+b_{16} \geq 25 \\
b_{37}+b_{17} \geq 26 \\
b_{38}+b_{18} \geq 26\end{array}$ & $\begin{array}{l}b_{22}+b_{48} \geq 25 \\
b_{23}+b_{49} \geq 25 \\
b_{24}+b_{50} \geq 24 \\
b_{26}+b_{51} \geq 24 \\
b_{26}+b_{52} \geq 23 \\
b_{36}+b_{13} \geq 24 \\
b_{37}+b_{14} \geq 25 \\
b_{38}+b_{15} \geq 25\end{array}$ & $\begin{array}{l}b_{19}+b_{39} \geq 26 \\
b_{20}+b_{40} \geq 26 \\
b_{21}+b_{41} \geq 26 \\
b_{27}+b_{47} \geq 25\end{array}$ \\
\hline
\end{tabular}

DLP BOUNDS ON THE BRANCH-COMPLEXITY OF THE $(64,39,10)$ BCH CODE

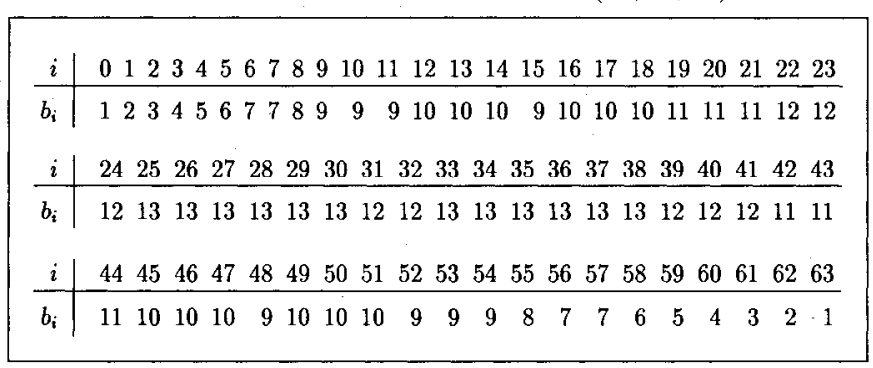

TABLE V

SOME CONSTRAINTS ON THE BRANCH-COMPLEXITY of THE $(64,39,10)$ BCH CODE

TABLE VI

New Bounds on the BRANCH-COMPLEXITY OF THE $(64,39,10)$ BCH CODE

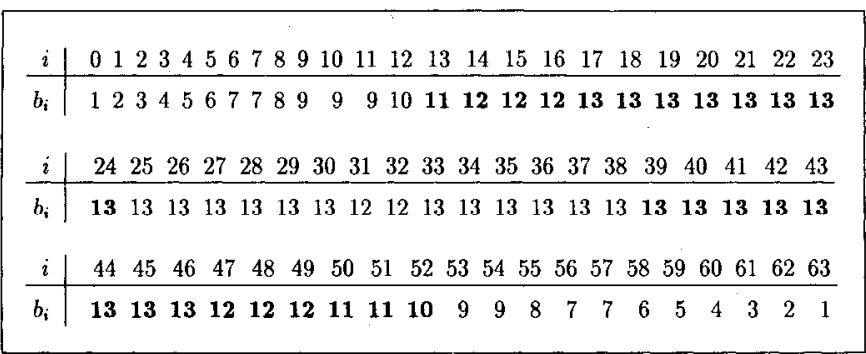

\section{B. Bounds Based on the Distance Set of the Code}

Theorem 5.2 is similar to Theorems 2.3, 3.1, and 4.1, in that it is based on the idea of partitioning the trellis into (more than two) sections, which is used in the foregoing subsection to obtain lower bounds on $E(C)$. We now show that the general ideas developed in Section III-B, based on the distance set and other combinatorial properties of the code, can be also employed to derive lower bounds on $E(C)$ which are often tighter than the DLP bound of (31). The application of these ideas, and in particular Proposition 3.5, to lower-bounding edge-complexity is best explained by means of specific examples.

The following two observations will be quite useful in the examples that follow. First, for binary linear codes, there are only four possible ways to connect the states in $S_{i}$ to the states in $S_{i+1}$, depending upon the values of $f_{i}-f_{i+1}$ and $p_{i+1}-p_{i}$. This observation was first made in [24], and was also noted independently in [20]. The four general types of branch structure are summarized in Table VII, and will be denoted by the mnemonics $=,<$,$\rangle , and \bowtie$ respectively.

Second, for linear codes, the subcodes $\mathcal{P}_{\sigma}$ and $\mathcal{F}_{\sigma}$ in Proposition 3.5 may be identified with cosets of the past and future subcodes $\mathcal{P}_{i}$ and $\mathcal{F}_{i}$, respectively. As a consequence, 
TABLE VII

The Four Basic Types of Trellis Structure

\begin{tabular}{|c|c|c|c|c|c|}
\hline \multirow{2}{*}{$\Delta f_{i}$} & \multirow{2}{*}{$\Delta p_{i}$} & \multirow{2}{*}{$\begin{array}{l}\text { Branch } \\
\text { structure }\end{array}$} & \multirow{2}{*}{$\begin{array}{l}\text { Branch } \\
\text { complexity }\end{array}$} & \multicolumn{2}{|c|}{ State structure } \\
\hline & & & & $S_{i}$ & $S_{i+1}$ \\
\hline 0 & 0 & & $b_{i}=s_{i}=s_{i+1}$ & & $\rightarrow$ \\
\hline 0 & 1 & & $b_{i}=s_{i}=s_{i+1}+1$ & $\underset{0}{\infty}$ & L \\
\hline & 0 & & $b_{i}=s_{i}+1=s_{i+1}$ & & $\underline{\epsilon}$ \\
\hline & 1 & & $b_{i}=s_{i}+1=s_{i+1}+1$ & $>$ & $\sum_{\infty}^{\infty}$ \\
\hline
\end{tabular}

we have

Lemma 5.3: For any $\sigma \in S_{i}$

$$
\begin{aligned}
& \log _{q}|P(\phi, \sigma)|=\operatorname{dim} \mathcal{P}_{\sigma}=p_{i} \\
& \log _{q}|P(\sigma, \varphi)|=\operatorname{dim} \mathcal{F}_{\sigma}=f_{i}
\end{aligned}
$$

Lemma 5.3 will be used extensively in the following examples.

Example 5.2: Let $C$ be the $(15,5,7) \mathrm{BCH}$ code with dual distance 4 . A trellis with 156 edges for this code was found in [8], and the DLP bound on edge-complexity is $E(C) \geq 148$ (see [8, Table 1]). We now close the gap between these bounds, establishing $E(C)=156$. The DLP bounds on the state and branch complexity profiles are given by

\begin{tabular}{|c|c|c|c|c|c|c|c|c|c|c|}
\hline$i$ & 8 & 9 & 10 & 11 & & 12 & 13 & & 14 & 15 \\
\hline$s_{i}$ & 3 & 4 & 4 & 3 & & 3 & 2 & & 1 & 0 \\
\hline$b_{i}$ & 4 & 4 & & & 3 & & 3 & 2 & & \\
\hline & $<$ & $=$ & & & $=$ & & $>$ & $>$ & & \\
\hline
\end{tabular}

\begin{tabular}{l|llllllllllll}
$i$ & 0 & & 1 & & 2 & & 3 & 4 & 5 & 6 & 7 & 8 \\
\hline$s_{i}$ & 0 & & 1 & & 2 & & 3 & 3 & 4 & 4 & 3 & 3 \\
$b_{i}$ & & 1 & & 2 & & 3 & 3 & 4 & 4 & 4 & 3 & \\
& & $<$ & $<$ & $<$ & $=$ & $<$ & $=$ & $>$ & $=$
\end{tabular}

Assume that there exists a trellis for $C$ which attains the branch-complexity profile in (35). This assumption, along with the lower bounds on $s_{i}$ which must be satisfied in any trellis for $C$, uniquely determines the branch structure at each position, which is also shown in (35). In particular, it follows that all the states in $S_{7}$ are of the type $>-$ Let $\sigma$ be such a state. Then $\left|\mathcal{P}_{\sigma}\right| \geq 2$. Since we also have $\log _{2}\left|\mathcal{P}_{\sigma}\right|=p_{7} \leq$ $k(7 ; C)=1$ by Lemma 5.3 , it is easy to see that $\left|\mathcal{P}_{\sigma}\right|=2$ and that $D\left(\mathcal{P}_{\sigma}\right)=\{0,7\}$. The distance set of $C$ is given by $D(C)=\{0,7,8,15\}$, and we conclude by Proposition 3.5 that

$$
\begin{aligned}
D\left(\mathcal{F}_{\sigma}\right) & \subset D(C) \Theta D\left(\mathcal{P}_{\sigma}\right) \\
& =\{0,7,8,15\} \cap\{-7,0,1,8\}=\{0,8\}
\end{aligned}
$$

However, referring to the branch structure in (35), we see that $P(\sigma, \varphi)$ contains two paths which must coincide at position $i=7$. Therefore, the distance between these two paths is at most $15-8=7$. This contradicts (36) and shows that a trellis attaining the branch-complexity profile of (35) does not exist. Hence, at least one of the values $b_{0}, b_{1}, \cdots, b_{14}$ must be strictly greater than the DLP bound thereupon. Clearly $b_{0}=b_{14}=1$ and $b_{1}=b_{13}=2$ have the maximum possible values for

\begin{tabular}{|c|c|c|c|c|c|c|c|c|c|c|c|}
\hline$i$ & 0 & 1 & 2 & 3 & & 4 & 5 & 6 & & & 8 \\
\hline & 0 & 1 & 2 & 3 & & 3 & 4 & 4 & & & \\
\hline$b_{i}$ & 1 & 2 & 3 & & 3 & 4 & 4 & & 4 & & \\
\hline & $<$ & $<$ & $<$ & & $=$ & $<$ & $=$ & & $=$ & & \\
\hline
\end{tabular}
their positions. Increasing the value of any other $b_{i}$ in (35) establishes $E(C) \geq 156$. In fact, it is easy to see that the optimal trellis for $C$ has the following structure:

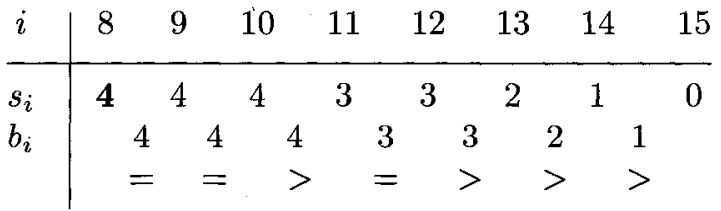

with the deviations from DLP bound set in bold face. This

\begin{tabular}{|c|c|c|c|c|c|c|c|c|c|c|c|}
\hline$i$ & 0 & 1 & 2 & 3 & 4 & & 5 & 6 & & & 8 \\
\hline$s$ & 0 & 1 & 2 & 3 & 3 & & 3 & 4 & & & 4 \\
\hline \multirow[t]{2}{*}{$b_{i}$} & 1 & 2 & 3 & 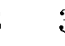 & & 4 & 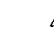 & 4 & 4 & & 5 \\
\hline & $<$ & $<$ & $<$ & $=$ & & $\bowtie$ & 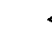 & & $=$ & & \\
\hline
\end{tabular}
indeed gives $E(C)=156$.

\begin{tabular}{|c|c|c|c|c|c|c|c|c|c|}
\hline$i$ & 8 & 9 & 10 & 11 & 12 & & & & \\
\hline$s$ & 4 & 4 & 3 & 3 & 3 & & & 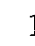 & \\
\hline$b_{i}$ & 4 & 4 & 4 & & 3 & 3 & & & 1 \\
\hline & $=$ & $>$ & $\bowtie$ & & $=$ & $>$ & & & $>$ \\
\hline
\end{tabular}

Example 5.3: Now let $C$ be the $(15,7,5) \mathrm{BCH}$ code. The DLP bounds on the branch and state complexity are

and the corresponding bound on the edge-complexity is $E(C) \geq 172$, as in [8, Table 1]. We now show that $s_{8} \geq 5$ for this code. Assume to the contrary that $s_{8}=7-p_{8}-f_{8}=4$. Since $p_{8} \leq K(8,5)=2$ and $f_{8} \leq K(7,5)=1$, this implies $p_{8}=2$ and $f_{8}=1$. Hence for all $\sigma \in S_{8}, \mathcal{P}_{\sigma}$ is a $(8,2,5)$ code and $\mathcal{F}_{\sigma}$ is a $(7,1,5)$ code. The $(8,2,5)$ linear code is unique and has distance set $D\left(\mathcal{P}_{\sigma}\right)=\{0,5,6\}$. (Note that the distance set of a code, as opposed to the weight distribution or the weight set, is invariant under translations.) The distance set of $C$ is given by $D(C)=\{0,5,6,7,8,9,10,15\}$, and thus

$$
\begin{aligned}
D\left(\mathcal{F}_{\sigma}\right) & \subset D(C) \Theta D\left(\mathcal{P}_{\sigma}\right) \\
& =\{0,5,6,7,8,9,10,15\} \cap\{-5,0,1,2,3,4,5,10\} \\
& \quad \cap\{-6,-1,0,1,2,3,4,9\} \\
& =\{0\} .
\end{aligned}
$$

This shows that if $p_{8}=2$ then $f_{8}=0$, and establishes $s_{8} \geq 5$. By a symmetric argument $s_{7}=s_{15-8} \geq 5$ as well. Hence $b_{6} \geq s_{7} \geq 5$ and $b_{8} \geq s_{8} \geq 5$, which implies $E(C) \geq 204$. $\diamond$ 
Example 5.4: Consider the $(31,10,12) \mathrm{BCH}$ code with dual distance 5 . The DLP bounds for this code are

\begin{tabular}{l|l|lllllllllllllll}
$i$ & 0 & 1 & 2 & 3 & 4 & 5 & 6 & 7 & 8 & 9 & 10 & 11 & 12 & 13 & 14 & 15 \\
\hline$b_{i}$ & 1 & 2 & 3 & 4 & 4 & 5 & 6 & 6 & 7 & 7 & 8 & 8 & 7 & 8 & 8 & 8 \\
$i$ & 16 & 17 & 18 & 19 & 20 & 21 & 22 & 23 & 24 & 25 & 26 & 27 & 28 & 29 & 30 \\
\hline$b_{i}$ & 8 & 8 & 7 & 8 & 8 & 7 & 7 & 6 & 6 & 5 & 4 & 4 & 3 & 2 & 1
\end{tabular}

and therefore $E(C) \geq 3484$ (cf. [8]). Consider positions $i=12,13, \cdots, 17$. Clearly $p_{i} \leq K(i, 12)=1$ at these positions, and hence $\mathcal{P}_{\sigma}$ is a code of dimension at most 1 for any $\sigma \in S_{i}$. The distance set of this code is given in $\left[27\right.$, p. 255] as $D(C)=\{0,12,16,20,31\}$. Thus if $p_{i}=1$ then the possible distance sets for $P_{\sigma}$ are $\{0,12\},\{0,16\}$, and $\{0,12,16\}$. In each of the three cases we get by Proposition 3.5 that

$$
D\left(\mathcal{F}_{\sigma}\right) \subset D(C) \ominus D\left(\mathcal{P}_{\sigma}\right)=\{0\} .
$$

Hence $p_{i}=1$ implies $f_{i}=0$, and therefore also $f_{i+1}=0$. Now $b_{i} \geq 10-p_{i}-f_{i+1}$, and by the foregoing argument this reduces to

$$
b_{i} \geq \min \left\{9,10-f_{i+1}\right\} \geq \min \{9,10-K(30-i, 12)\}
$$

for $i=12,13, \cdots, 17$. Equation (37) gives the following bounds on the branch complexity profile:

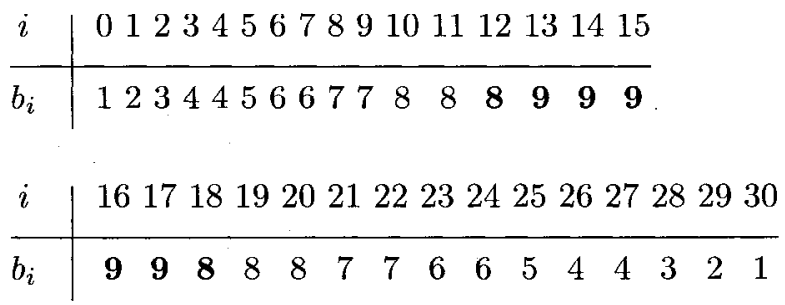

where the improvement at position 18 follows by symmetry. The resulting lower bound on edge-complexity is $E(C) \geq$ 5020 .

Example 5.5 Our final example is the $(16,256,6)$ nonlinear Nordstrom-Robinson code. The trellises for $\mathcal{N}_{16}$ given in [11] and [36] have the following profiles

\begin{tabular}{c|cccccccccc}
$i$ & 0 & 1 & 2 & 3 & 4 & 5 & 6 & 7 & 8 \\
\hline$\left|S_{i}\right|$ & 1 & 2 & 4 & 8 & 16 & 32 & 64 & 128 & 64 \\
$\left|\mathcal{B}_{i}\right|$ & & 2 & 4 & 8 & 16 & 32 & 64 & 128 & 128 & \\
& & $<$ & $<$ & $<$ & $<$ & $<$ & $<$ & $<$ & $>$ &
\end{tabular},

\begin{tabular}{c|cccccccccc}
$i$ & 8 & 9 & 10 & 11 & 12 & 13 & 14 & 15 & 16 \\
\hline$\left|S_{i}\right|$ & 64 & 128 & 64 & 32 & 16 & 8 & 4 & 2 & 1 \\
$\left|\mathcal{B}_{i}\right|$ & \multicolumn{1}{c|}{128} & 128 & 64 & 32 & 16 & 8 & 4 & 2 & \\
& $<$ & $>$ & $>$ & $>$ & $>$ & $>$ & $>$ & $>$
\end{tabular}

with the structure shown shared by all the states. The lower bound of (25) on the state-complexity of $\mathcal{N}_{16}$, along with Proposition 3.3, show that this profile is optimal at positions $0,1,2,3,4,5$ and $10,11,12,13,14,15$. Furthermore, $\left|\mathcal{B}_{6}\right| \geq$ $\left|S_{7}\right| \geq 96$ and $\left|\mathcal{B}_{9}\right| \geq\left|S_{9}\right| \geq 96$ by Corollary 3.6. Corollary 3.6 also implies $\left|\mathcal{B}_{7}\right|+\left|\mathcal{B}_{8}\right| \geq\left|S_{7}\right|+\left|S_{9}\right| \geq 192$. However, we now deduce a tighter lower bound on $\left|\mathcal{B}_{7}\right|+\left|\mathcal{B}_{8}\right|$. To this end, all the states $\sigma \in S_{8}$ may be partitioned into the following types:

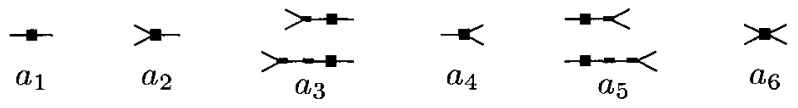

depending on the number and position of merges and expansions in the path(s) passing through $\sigma$ (the state $\sigma$ itself is denoted by the larger $\boldsymbol{m}$ above). The letters $a_{1}, a_{2}, \cdots, a_{6}$ indicate the number of states of each type. To see that these are the only possible kinds of states, apply Propositions 3.3, 3.4, and 3.5. Now, evidently

$$
\left|\mathcal{B}_{7}\right|+\left|\mathcal{B}_{8}\right|=2 a_{1}+3 a_{2}+2 a_{3}+3 a_{4}+2 a_{5}+4 a_{6}
$$

while counting the total number of paths in the trellis, with $i=8$ in (21), we obtain

$$
256=a_{1}+2 a_{2}+2 a_{3}+2 a_{4}+2 a_{5}+4 a_{6} .
$$

It follows that

$$
\left|\mathcal{B}_{7}\right|+\left|\mathcal{B}_{8}\right|=256+a_{1}+a_{2}+a_{4} \geq 256 .
$$

Referring to (38), we see that this bound is optimal, so that $\left|\mathcal{B}_{7}\right|+\left|\mathcal{B}_{8}\right|=256$. Combining all of the foregoing results, gives

$$
700 \leq E\left(\mathcal{N}_{16}\right) \leq 764 .
$$

We conjecture that $E\left(\mathcal{N}_{16}\right)=764$, and that the optimal trellis is given by (38).

We note that in all the foregoing examples, the technique of Section V-A fails to produce an improvement over the DLP bound-there are no useful constraints. In general, however, the results of the two subsections can be easily combined: the lower bounds on $b_{i}$ derived using Proposition 3.5 (or any other method) can be incorporated as additional constraints in the minimization problem of Theorem 5.2.

The lower bounds for all the codes considered in this section, along with many more examples, are summarized in Table VIII. The DLP bounds for the $(16,7,6) \mathrm{BCH},(18,9,5)$ quadratic residue, and $(31,10,12) \mathrm{JMG}$ codes (see Berlekamp [3] for the reference to the latter) were given in the table of [8]. The improved lower bounds for these codes follow by applying Proposition 3.5 in a manner similar to the foregoing examples. All the other lower bounds require the use of Theorem 5.2. All the upper bounds are from [8].

\section{ASYMPTOTIC BOUNDS ON TRELLIS COMPLEXITY}

In this section we investigate the asymptotic behavior of the new lower bounds on state-complexity of linear and nonlinear codes, derived in Sections II and III of this paper. In particular, we will be interested in the relative trellis complexity $\varsigma=s / n$ as $n \rightarrow \infty$. Our earlier results [24], [25] show that $\varsigma$ is strictly greater than zero for any asymptotically good sequence of codes with rate fixed at $R$ and relative minimum distance $d / n$ fixed at $\delta$. In fact, it is easy to see that the lower bound of 
TABLE VIII

LOWER Bounds on Trellis EDGe-Complexity for SOME Binary Codes

\begin{tabular}{|c|c|c|c|c|}
\hline \multirow{2}{*}{ Code } & \multicolumn{4}{|c|}{ Bounds on $E(C)$} \\
\hline & $\underset{\text { bound }}{\text { DLP }}$ & $\begin{array}{c}\text { New } \\
\text { bound }\end{array}$ & $\begin{array}{l}\text { Upper } \\
\text { bound }\end{array}$ & Comments \\
\hline 1. $\mathrm{BCH}[15,5,7]$ & 148 & 156 & 156 & Example 5.2 \\
\hline 2. $\mathrm{BCH}[15,7,5]$ & 172 & 204 & 300 & Example 5.3 \\
\hline 3. BCH $[16,7,6]$ & 236 & 332 & 420 & Table 1 of $[8]$ \\
\hline 4. $\mathrm{NR}(16,256,6)$ & 402 & 700 & 764 & Example 5.5 \\
\hline 5. Hamming $[18,10,4]$ & 46 & 93 & & \\
\hline 6. $\mathrm{QR}[18,9,6]$ & 508 & 572 & 732 & Table 1 of $[8]$ \\
\hline 7. $\mathrm{BCH}[31,10,12]$ & 3484 & 5020 & 7068 & Example 5.4 \\
\hline 8. JMG $[31,10,12]$ & 3484 & 5020 & 7500 & Table 1 of $[8]$ \\
\hline 9. Hamming $[41,32,4]$ & 320 & 576 & & \\
\hline 10. Shearer $[44,29,6]$ & 1458 & 3358 & & \\
\hline 11. $\mathrm{BCH}[64,39,10]$ & 161020 & 274172 & 95839228 & Example 5.1 \\
\hline 12. $\mathrm{BCH}[70,45,9]$ & 79270 & 196006 & & \\
\hline 13. $\mathrm{BCH}^{*}[73,46,10]$ & 87592 & 273960 & & \\
\hline 14. $\mathrm{BCH}[76,44,11]$ & 71004 & 424088 & & \\
\hline 15. $\mathrm{BCH}[76,50,9]$ & 65818 & 347478 & & \\
\hline 16. Alternant* $[82,55,9]$ & 53970 & 96984 & & \\
\hline 17. Goppa $[97,62,12]$ & 221804 & 705132 & & \\
\hline 18. Goppa $[105,56,16]$ & 490276 & 3590948 & & \\
\hline 19. Goppa $*[115,87,9]$ & 291366 & 619046 & & \\
\hline 20. Goppa* $[126,110,6]$ & 85152 & 101536 & & \\
\hline 21. $\mathrm{BCH}[127,78,15]$ & 1028702 & 7221854 & & \\
\hline 22. $\mathrm{BCH}[127,85,13]$ & 1029664 & 9287200 & & \\
\hline 23. $\mathrm{BCH}[127,92,11]$ & 781866 & 2629162 & & \\
\hline 24. $\mathrm{BCH}[127,99,9]$ & 633444 & 1124964 & & \\
\hline
\end{tabular}

(5) translates into ${ }^{6}$

$$
\varsigma \gtrsim \delta R
$$

for $n \rightarrow \infty$. In addition, in the recent work of Zyablov and Sidorenko [42] the authors extend Muder's bound (2) to asymptotics, cstablishing that

$$
\varsigma \gtrsim R-R_{\max }(2 \delta)
$$

where $R_{\max }(\cdot)$ is the McEliece-Rodemich-Rumsey-Welch [31] upper bound, given by

$$
\begin{aligned}
R_{\max }(\delta)=\min _{1-2 \delta \leq u \leq 1}\{ & 1+H_{2}\left(1 / 2-1 / 2 \sqrt{1-u^{2}}\right) \\
& \left.-H_{2}\left(1 / 2-1 / 2 \sqrt{u^{2}+2 u \delta+2 \delta}\right)\right\}
\end{aligned}
$$

for $0 \leq \delta \leq 0.5$. Equations (39) and (40) are presently the best known lower bounds on $\varsigma$.

We note that the asymptotic bounds on branch and state complexities coincide, in view of the obvious relation

$$
s \leq b \leq s+1
$$

\footnotetext{
${ }^{6}$ The notation $\gtrsim$ and $\lesssim$ is employed herein to denote inequalities that hold asymptotically for $n \rightarrow \infty$. Thus $f(n) \gtrsim g(n)$ means $f(n) \geq$ $g(n)(1-o(1))$.
}

Furthermore, (41) also implies that

$$
E(C)=\sum_{i=0}^{n-1} q^{b_{i}} \leq n q^{s+1}
$$

and, therefore, the relative edge-complexity is bounded by

$$
\varsigma \leq \frac{\log _{q} E(C)}{n} \leq \varsigma+\frac{\log _{q} n+1}{n}
$$

Since $\left(\log _{q} n+1\right) / n$ vanishes as $n \rightarrow \infty$, we conclude that asymptotically edge-complexity is equivalent to statecomplexity. Indeed, other measures of trellis complexity introduced in [20], such as the total number of states, the total number of merges, the total span of the generator matrix, also coincide with $\varsigma$ at $n \rightarrow \infty$. We will therefore take $\varsigma=s / n$ as the single asymptotic measure of trellis complexity.

In deriving the asymptotic equivalent of Theorems 2.3 and 3.1 , we will restrict ourselves to partitions into sections of equal length. We will then show that such partitions are indeed asymptotically optimal. The following theorem holds for linear as well as nonlinear codes described by one-to-one trellises.

Theorem 6.1: For all $L=2,3, \cdots$

$$
\varsigma \gtrsim \frac{R-R_{\max }(L \delta)}{L-1} .
$$


Proof: By Theorem 3.1 we have that

$$
\begin{aligned}
\varsigma \geq \frac{1}{L-1}\left(\frac{\log _{q}|C|}{n}-\frac{1}{n} \sum_{j=1}^{L} \log _{q} M\left(l_{j} ; C\right)\right) \\
=\frac{R-\frac{1}{n} \sum_{j=1}^{L} \log _{q} M\left(l_{j} ; C\right)}{L-1}
\end{aligned}
$$

for any fixed $n$ and $L$. Thus it would suffice to exhibit a partition $l_{1}, l_{2}, \cdots l_{L}$ of the trellis, such that

$$
\frac{1}{n} \sum_{j=1}^{L} \log _{q} M\left(l_{j} ; C\right) \lesssim R_{\max }(L \delta) .
$$

Denote $\eta=\lfloor n / L\rfloor$ and choose the section lengths so that $l_{j} \leq \eta+1$ for all $j=1,2, \cdots, L$. Then, using the fact that $\log _{q} M\left(l_{j} ; C\right) \leq \log _{q} M\left(l_{j}-1 ; C\right)+1$, we have

$$
\begin{aligned}
\frac{1}{n} \sum_{j=1}^{L} \log _{q} M\left(l_{j} ; C\right) & \leq \frac{1}{n}\left(L \log _{q} A_{q}(\eta, d)+L\right) \\
& \leq \frac{\log _{q} \Lambda_{q}(\eta, d)}{\eta}+\frac{L}{n}
\end{aligned}
$$

where $A_{q}(n, d)$ is the largest possible number of codewords in a code of length $n$ and minimum distance $d$ over an alphabet of size $q$. Evidently,

$$
\frac{\log _{q} A_{q}(\eta, d)}{\eta} \lesssim R_{\max }(d / \eta) \leq R_{\max }(L \delta)
$$

where the second inequality follows from the fact that $R_{\max }(\cdot)$ is a nonincreasing function. Now, for any fixed $L$, the term $L / n$ in (43) clearly vanishes when $n \rightarrow \infty$. This establishes (42) and completes the proof of the theorem.

We now show that the partition into sections of (asymptotically) equal length, employed in Theorem 6.1 , indeed produces the tightest possible lower bound on $\varsigma$. This essentially follows from the $U$-convexity of the function $R_{\text {n1ax }}(\cdot)$. Suppose that the trellis is partitioned into $L$ sections of lengths $l_{j}=\alpha_{j} n$, where $\alpha_{1}, \alpha_{2}, \cdots, \alpha_{L}>0$ are real constants with

$$
\alpha_{1}+\alpha_{2}+\cdots+\alpha_{L}=1 \text {. }
$$

Going through the proof of Theorem 6.1 , it can be readily verified that this produces the lower bound

$$
\varsigma \gtrsim \frac{1}{L-1}\left(R-\sum_{j=1}^{L} \alpha_{j} R_{\max }\left(\delta / \alpha_{j}\right)\right) .
$$

Now, observe that the $\cup$-convexity of $R_{\max }(\cdot)$ implies that

$$
\begin{aligned}
& R_{\max }\left(\alpha_{1} x_{1}+\alpha_{2} x_{2}+\cdots+\alpha_{L} x_{L}\right) \\
& \quad \leq \alpha_{1} R_{\max }\left(x_{1}\right)+\alpha_{2} R_{\max }\left(x_{2}\right)+\cdots+\alpha_{L} R_{\max }\left(x_{L}\right)
\end{aligned}
$$

for any $x_{1}, x_{2}, \cdots, x_{L}$. Taking $x_{j}=\delta / \alpha_{j}$ in the above expression, we obtain

$$
R_{\max }(L \delta) \leq \sum_{j=1}^{L} \alpha_{j} R_{\max }\left(\delta / \alpha_{j}\right)
$$

which shows that the lower bound of (44) is never stronger than Theorem 6.1. Indeed, a similar $U$-convexity argument was employed by Zyablov and Sidorenko [42] to derive their asymptotic bound.

Theorem 6.1 produces a countably infinite family of lower bounds on $\varsigma$, and it is easy to see that the apparently dissimilar bounds of (39) and (40) are in fact the extreme members of this family corresponding to $L-2$ and $L \simeq 1 / \delta$, respectively. In the latter case, Theorem 6.1 reduces to

$$
\varsigma \gtrsim \delta R-\delta R_{\max }(1)=\delta R .
$$

In fact, this bound can be now readily improved to

$$
\varsigma \gtrsim 2 \delta R
$$

by taking $L \simeq 1 / 2 \delta$ and observing that $R_{\max }(u)=0$ for $u \geq 0.5$ by the Plotkin bound. The relationship between the lower bounds on 5 corresponding to $L=2,3,4,5$ and $L \simeq 1 / 2 \delta$ is illustrated in Fig. 1 for the binary codes meeting the Gilbert-Varshamov bound.

Although this is not apparent from Fig. 1, we note that there exist values of $R$ and $\delta$ on the curve $R=1-H_{2}(\delta)$ described by the Gilbert-Varshamov bound, for which the lower bound $\varsigma \gtrsim 2 \delta R$ is stronger that the bound obtained by taking any fixed value of $L$ in Theorem 6.1. The proof of this statement is rather elaborate, and is therefore omitted. Observe, however, that these values of $R, \delta$ lie in the neighborhood of the point $R=1$ and $\delta=0$. Thus in a sense, the infinite family of bounds in Theorem 6.1 converges to (45) as $R \rightarrow 1$, and to (40) as $R \rightarrow 0$.

The best known upper bound on the trellis complexity of binary codes meeting the Gilbert-Varshamov bound is due to Kudryashov and Zakharova [23]. In this paper, the authors use the results of Barg and Dumer [2], along with ingenious construction of partially tail-biting trellises (cf. [26]), to show that there exist codes on the Gilbert-Varshamov curve $R=$ $1-H_{2}(\delta)$, whose trellis complexity satisfies

$$
\varsigma \lesssim \begin{cases}1-H_{2}(\delta), & 0 \leq R \leq 1-H_{2}(1 / 1) \\ H_{2}(2 \delta)-H_{2}(\delta), & 1-H_{2}(1 / 1) \leq R \leq 1 .\end{cases}
$$

The resulting upper bound on $\varsigma$ is illustrated in Fig. 2. It follows from our results combined with those of [23] that the trellis complexity of the best binary codes lies somewhere in the shaded area of Fig. 2.

\section{Note Added in Proof:}

We have been recently informed that the upper bound of (46) on the trellis complexity of binary codes meeting the Gilbert-Varshamov bound (see also Fig. 2), which we attributed here to Kudryashov and Zakharova [23], was first obtained by I. I. Dumer in [43]. The later work of Kudryashov and Zakharova [23], however, contains a different construction and proof of this bound.

\section{ACKNOWLEDGMENT}

The authors wish to thank G. D. Forney, Jr. for very helpful discussions. We are also grateful to A. E. Brouwer for access 
to the on-line version of [5], to J. B. Shearer for providing the parity-check matrices of his codes, and to I. F. Blakc, S. Lin, F. Kschischang, and R. J. McEliece for preprints of their papers.

\section{REFERENCES}

[1] L. R. Bahl, J. Cocke, F. Jelinek, and J. Raviv, "Optimal decoding of lincar codes for minimizing symbol crror rate," IEEE Trans. Inform. Theory, vol. IT-20, pp. 284-287, 1974.

[2] A. M. Barg and I. I. Dumer, "Concatenated decoding algorithms with partial enumeration of code vectors," Probl. Peredachi Inform., vol. 22, pp. 3-10, 1986 (in Russian)

[3] E. R. Berlekamp, Algebraic Coding Theory. New York: McGraw-Hill, 1968.

[4] D. P. Bertsekas, Constrained Optimization and Lagrange Multiplier Methods. New York: Academic Press, 1982.

[5] A. E. Brouwer and T. Verhoeff, "An updated table of minimum-distance bounds for binary linear codes," IEEE Trans. Inform. Theory, vol. 39, pp. $662-677,1993$.

[6] G. Cohen, S. Litsyn, and G. Zémor, "Upper bounds on generalized distances," IEEE Trans. Inform. Theory, vol. 40, pp. 2090-2092, 1994.

[7] J. H. Conway and N. J. A. Sloane, Sphere Packings, Lattices and Groups. New York: Springer-Verlag, 1988

[8] S. Dolinar, L. Ekroot, A. B. Kiely, R. J. McEliece, and W. Lin, "The permutation trellis complexity of linear block codes," in Proc. 32nd Allerton Conf. on Communications, Control, and Computing (Monticello, IL., Sept. 1994), pp. 60-74.

[9] G. D. Forney, Jr., "The Viterbi algorithm," Proc. IEEE, vol. 61, pp. 268-278, 1973

[10] - "Coset codes II: Binary lattices and related codes," IEEE Trans. Inform. Theory, vol. 34, pp. 1152-1187, 1988.

[11] __ "Dimension/length profiles and trellis complexity of linear block codes," IEEE Trans. Inform. Theory, vol. 40, pp. 1741-1752, 1994.

[12] __ "Density/length profiles and trellis complexity of lattices," IEEE Trans. Inform. Theory, vol. 40, pp. 1752-1772, 1994.

[13] G. D. Forney, Jr. and M. D. Trott, "The dynamics of group codes: State spaces, trellis diagrams and canonical encoders," IEEE Trans. Inform Theory, vol. 39, pp. 1491-1513, 1993.

[14] T. Fujiwara, T. Kasami, R. Morelos-Zaragoza, and S. Lin, "The state complexity of trellis diagrams for a class of generalized concatenated codes," preprint.

[15] V. D. Goppa, "Bounds for codes," Dokl. Akad. Nauk, vol. 333, p. 423, 1993 (in Russian)

[16] T. Helleseth, T. Kløve, and J. Mykkelveit, "The weight distribution of irreducible cyclic codes," Discr. Math., vol. 18, pp. 179-211, 1977.

[17] T. Helleseth, T. Kløve, and Ø. Ytrehus, "Generalized Hamming weights of linear codes," IEEE Trans. Inform. Theory, vol. 38, pp. 1133-1140, 1992.

[18] 'T. Kasami, T. Takata, T. Fujiwara, and S. Lin, "On complexity of trellis structure of linear block codes," presented at the IEEE Int. Symp. Inform. Theory, Budapest, Hungary, 1991.

[19] "On the optimum bit orders with respect to the state complexity of trellis diagrams for binary linear codes," IEEE Trans. Inform. Theory, vol. 39, pp. 242-243, 1993.

[20] A. B. Kiely, S. Dolinar, R. J. McEliece, L. Ekroot, and W. Lin, "Trellis decoding complexity of linear block codes," IEEE Trans. Inform. Theory, submitted for publication.
[21] F. R. Kschischang and G. B. Horn, "A heuristic for ordering a linear block code to minimize trellis state complexity," in Proc. 32nd Allerton Conf. on Communication, Control, and Computing (Monticello, IL., Sept. 1994), pp. 75-84.

[22] F. R. Kschischang and V. Sorokine, "On the trellis structure of hlock codes," this issue, pp. 1924-1937.

[23] B. D. Kudryashov and T.G. Zakharova, "Block codes from convolutional codes," Probl. Peredachi Inform., vol. 25, pp. 98-102, 1989 (in Russian).

[24] A. Lafourcade and A. Vardy, "On trellis complexity of good codes," in Proc. 28th Annual Conf. on Information Sciences and Systems (Princeton, NJ, Mar. 1994)

[25] _ "Asymptotically good codes have infinite trellis complexity," IEEE Trans. Inform. Theory, vol. 41, pp. 555-559, 1995.

[26] H. H. Ma and J. K. Wolf, "On tail biting convolutional codes," IEEE Trans. Commun., vol. COM-34, pp. 104-111, 1986.

[27] F. J. MacWilliams and N. J. A. Sloane, The Theory of Error-Correcting Codes. New York: North-Holland, 1977.

[28] J. L. Massey, "Foundation and methods of channel encoding," in Proc. Int. Conf. on Information Theory and Systems, NTG-Fachberichte, Berlin, 1978

[29] R. J. McEliece, "The Viterbi decoding complexity of linear block codes," presented at the IEEE Int. Symp. Inform. Theory, Trondheim, Norway, 1994.

[30] _ "On the BCJR trellis for linear block codes," IEEE Trans. Inform. Theory, submitted for publication.

[31] R. J. McEliece, E. R. Rodemich, H. C. Rumsey, and L. R. Welch, "New upper bounds on the rate of a code via the Delsarte-MacWilliams inequalities,"' IEEE Trans. Inform. Theory, vol. IT-23, pp. 157-166, 1977

[32] D. J. Muder, "Minimal trellises for block codes," IEEE Trans. Inform. Theory, vol. 34, pp. 1049-1053, 1988.

[33] J. B. Shearer, personal communication, 1994.

[34] V. Tarokh and I. F. Blake, "Decoding complexity versus coding gain of lattices," IEEE Trans. Inform. Theory, submitted for publication.

[35] A. Vardy, "Dynamical structure of block codes," presented at the IEEE Int. Workshop on Coding, System Theory, and Symbolic Dynamics, Mansfield, MA, Oct. 1993.

[36] _ "The Nordstrom-Robinson code: Representation over GF(4) and efficient decoding," IEEE Trans. Inform. Theory, vol. 40, pp. 1686-1693, 1994

[37] A Vardy and Y. Be'ery, "Maximum-likelihood soft decision decoding of BCH codes," IEEE Trans. Inform. Theory, vol. 40, pp. 546-554, 1994.

[38] V. K. Wei, "Generalized Hamming weights for linear codes," IEEE Trans. Inform. Theory, vol. 37, pp. 1412-1418, 1991.

[39] _ "A survey of results on generalized Hanming weights for linear codes," presented at the IEEE Inform. Theory Workshop, Sunoso, Japan, 1993.

[40] J. K. Wolf, "Efficient maximum-likelihood decoding of linear block codes using a trellis," IEEE Trans. Inform. Theory, vol. IT-24, pp. 76-80, 1978.

141] $\emptyset$. Ytrehus, "On the trellis complexity of certain binary linear block codes," IEEE Trans. Inform. Theory, vol. 41, pp. 559-560, 1995.

[42] V. V. Zyablov and V. R. Sidorenko, "Bounds on complexity of trellis decoding of linear block codes," Probl. Peredachi Inform., vol. 29, pp. 3-9, 1993 (in Russian).

[43] I. I. Dumer, "On complexity of maximum likelihood decoding of the best concatenated codes," in Proc. 8th All-Union Conf. on Coding Theory and Information Theory (Moscow-Kuibushev, 1981), pp. 66-69 (in Russian). 